\title{
Chapter 4 \\ Extended Non-destructive Testing for the Bondline Quality Assessment of Aircraft Composite Structures
}

\author{
Paweł H. Malinowski, Tomasz Wandowski, Wiesław M. Ostachowicz, \\ Maxime Sagnard, Laurent Berthe, Romain Ecault, Igor Solodov, \\ Damien Segur, and Marc Kreutzbruck
}

\begin{abstract}
We present the results of extended non-destructive testing (ENDT) methods for bond line quality assessment in adhesive joints. The results presented were derived for important application scenarios with regards to aircraft manufacturing and the in-service repair of composite structures. The electromechanical impedance (EMI), laser shock adhesion testing (LASAT), and nonlinear ultrasound scanning (NUS) were used on flat coupon samples, scarfed samples, and curved samples. The EMI method applied to the flat coupons showed some relation of the frequency shift to the level of contamination. For the curved samples, there was insufficient sensitivity to differentiate distinct levels of contamination, while for scarfed samples in most cases both detection and distinction were possible. The LASAT method gave good results for the coupon samples, which were also in accordance with the results of the $\mathrm{G}_{\mathrm{IC}}$ and $\mathrm{G}_{\mathrm{IIC}}$ tests. For coupon samples with multiple contaminations, we obtained results with varying significance. In the case of NUS, the measurements revealed an increase in nonlinearity affected by contamination at the interphase between the CFRP adherend and the adhesive layer for the majority of scenarios comprising single contamination of flat coupons and scarfed samples. The effect of multiple contaminations was a decrease in nonlinearity for the curved samples.
\end{abstract}

P. H. Malinowski $(\bowtie) \cdot$ T. Wandowski · W. M. Ostachowicz

Institute of Fluid-Flow Machinery, Polish Academy of Sciences, Fiszera 14, Gdańsk, Poland

e-mail: pmalinowski@imp.gda.pl

M. Sagnard · L. Berthe

PIMM, CNRS-ENSAM ParisTech, 151 Bd de l'Hôpital, 75013 Paris, France

R. Ecault

Airbus Operations S.A.S., 316 route de Bayonne, B.P. D4101, 31060 Cedex 9 Toulouse, France

I. Solodov $\cdot$ M. Kreutzbruck

Institut für Kunststofftechnik, Universität of Stuttgart, Pfaffenwaldring 32, 70569 Stuttgart,

Germany

D. Segur

CEA LIST, CEA Saclay, 91191 Gif-sur-Yvette, France

(C) The Author(s) 2021

W. Leite Cavalcanti et al. (eds.), Adhesive Bonding of Aircraft Composite Structures,

https://doi.org/10.1007/978-3-319-92810-4_4 
Keywords NDT $\cdot$ NDE $\cdot$ Adhesive bonds $\cdot$ Electromechanical impedance $\cdot$ Laser shock adhesion test $\cdot$ Nonlinear ultrasonics

\subsection{Introduction}

The merits of lightweight, high-strength composite materials characterized by unprecedented performance, energy efficiency, safety, and environmental compatibility are widely recognized and applied in many sectors of the economy. The role of technology in joining and consolidating composite parts and other materials is of primary importance to provide manufacturing and maintenance flexibility for highperformance composite components. In this context, the adequate non-destructive testing (NDT) and non-destructive evaluation (NDE) of interfacial adhesion, which determines the reliability of a structural bond, has long been considered a challenge for the NDT/E community [1].

This chapter presents methods for the assessment of the bond lines of aircraft composite structures, including a description thereof, as well as a discussion of the results obtained with each method. With regards to potentially suitable methods for the bond line quality assessment of aircraft composite structures, we have perceived a considerable advancement over the last decade. From a chronological standpoint, the research presented in this chapter is a continuation of the studies initiated within the framework of ENCOMB (extended non-destructive testing of composite bonds [2], an FP7 EU project. Originally, the idea behind ENCOMB was to screen assessment methods that could be utilized in standardized quality assurance procedures for bonded aerospace composite structures because, in essence, the NDT methods currently used in this field are neither sufficient nor reliable for the purpose of certifying bonded composite structures utilized in aerospace structures. This problem was initiated by the fact that more and more aerospace structures are being manufactured from fibre-reinforced composites, and aerospace engineers are looking for new methods to join these. The idea was to develop joining methods based on adhesive bonding, enabling a reduction of the aircraft weight through a reduction or even a total elimination of riveted joints, which are commonly used in aluminium-based aerospace structures. We would like to underline here that rivets are also common in aerospace composite structures, whereby they are joined in conjunction with an adhesive bond line in order to ensure the reliability of the joint in cases of bond line failure, an approach that is also referred to as "chicken rivets". Nowadays, according to the certification rules for aerospace structures, it is not possible to certify an aircraft composite structure that includes solely bonded joints (without additional rivets for safety). This is due to the continuing lack of reliable NDT methods and procedures for the certification of such structures.

The ENCOMB project screened NDT methods that are able to both assess the surface of composite structures prepared for adhesive bonding and evaluate weak bonds in composite structures. The process of adhesive bonding is applied to new composite parts at the manufacturing level or during a composite repair process. 
Improper preparation of the surface of the adherend (the composite part prepared for adhesive bonding) is one of the potential sources of a weak bond. Such problems can be caused by chemical contamination by hydraulic fluids (Skydrol) or kerosene during the repair process of a composite aerospace structure. The term weak bond refers to a bond line with reduced strength properties. Another source of weak bonds could be problems in the curing process of the adhesive caused by incorrect parameters, such as temperature. In ENCOMB project, typical scenarios for aerospace structures were selected and investigated, whereby small, flat carbon fibre reinforced polymer (CFRP) samples were utilized individually for surface contamination detection and bonded for bond line assessment. In each sample, only one type of contamination scenario was investigated. Subsequently, the appropriate NDT method was verified for new application areas related to the assessment of bond lines. Hereby, the following methods were investigated in the ENCOMB project: active thermography (vibrothermography and with optical excitation), THz/GHz reflectometry, nonlinear ultrasound, ultrasonic frequency analysis, laser ultrasound, laser scanning vibrometry, and electromechanical impedance. Some preliminary adaptations of NDT methods were made, and these adapted methods are referred to as extended non-destructive testing methods (ENDT).

In this chapter, we present selected results of the research related to the more recent and advanced ComBoNDT (quality assurance concepts for adhesive bonding of aircraft composite structures by advanced NDT) [3] H2020 EU project. This project is partially a continuation of the ENCOMB project; however, only the most promising ENDT methods from the ENCOMB project were further investigated, whereby the following methods are presented in this chapter: electromechanical impedance (EMI), laser shock adhesion testing (LASAT) and nonlinear ultrasound scanning (NUS). The research used small, flat CFRP samples with single contaminations, but further extended the study to include samples with combined contaminations, as well as curved samples and samples with scarfed bonding. Finally, components of real aerospace structures with stringers were also investigated, and the respective findings are reported in the next chapter.

The results presented in this chapter were achieved from the research conducted into the applied ENDT methods. Looking ahead, we would like to highlight that, in certain scenarios, these methods are sensitive to impacts affecting a bond strength reduction, and therefore, could potentially be utilized to identify not in order (NIO) joints during a bond line assessment.

\subsection{Electromechanical Impedance}

In this section, we will describe the principle and instrumentation of the electromechanical impedance (EMI) method and detail the results recently obtained in the ComBoNDT project. 


\subsubsection{Principle and Instrumentation}

The EMI technique utilizes measurements of the electrical parameters of a piezoelectric transducer bonded to the surface of the host structure. Due to the electromechanical coupling of the transducer and the structure, the mechanical characteristics of the structure have an influence on the electrical characteristics of the piezoelectric transducer. EMI is an active method. The transducer works both as an actuator by exciting the structure and as a sensor to sense the response in the defined frequency band. Data analysis for this method is performed in the frequency domain. Adhesive bonds have already been investigated with the EMI method by embedding a transducer into a lap joint $[4,5]$, these investigations were both numerical and experimental, but they have focused only on aluminium lap joints. Here, we investigate the EMI method in its application to the adhesive bonds of CFRP composites, whereby some of the initial results on this topic have been published in [6]. In the research reported here, the IM3570 laboratory impedance analyzer (Hioki) (Fig. 4.1a) was used and the focus was mostly on conductance spectra measured up to $5 \mathrm{MHz}$. The measurements were performed with a piezoelectric disc transducer that was bonded to the investigated sample with cyanoacrylate glue (Fig. 4.1b). The disc had a $10 \mathrm{~mm}$ diameter and a $0.5 \mathrm{~mm}$ thickness and was manufactured by CeramTec (SONOX P502 material). For the analysis, the root mean square value was calculated for the considered parts of the spectra, and the location and magnitude of the resonance peaks were also tracked.

\subsubsection{EMI Results}

\subsubsection{EMI Results on the Coupon Level Samples}

For the coupon samples, two groups of samples were investigated. The first group represented production-related contamination of the samples, while the second group represented repair-related modifications/contaminations of the samples. The sample set investigated for the production scenario comprised three reference samples, nine samples contaminated with a release agent, nine samples contaminated with moisture, nine samples contaminated with fingerprints (artificial sweat), and six samples with a combined contamination of release agent and fingerprints. The sensors were glued to the middle of the sample surface. After considering the registered spectra, some of the results were rejected (I-P-RE3, I-P-RA-1-2, I-P-RA-2-3, I-P-RA-3-2, I-PMO-2-2, I-P-FP-1-2, and I-P-FP-2-2) due to the different shapes of the spectra. In a previously reported study referring to samples with moisture, release agent, and fingerprint contamination, the bandwidth corresponding to the maximum peak of conductance was inspected [7], whereby the average values of RMS on differential spectra were also analyzed and no clear sensitivity to the contamination level was observed. In the research reported here, the measured spectra were inspected and a local resonance was identified for the bonded samples in the bandwidth 4.3-4.7 MHz. 
(a)

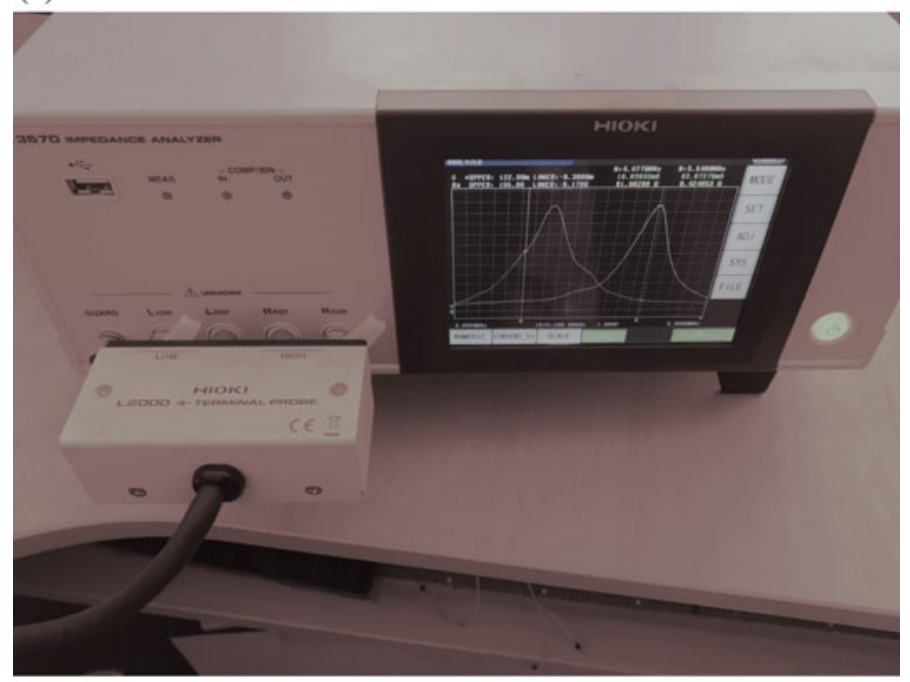

(b)

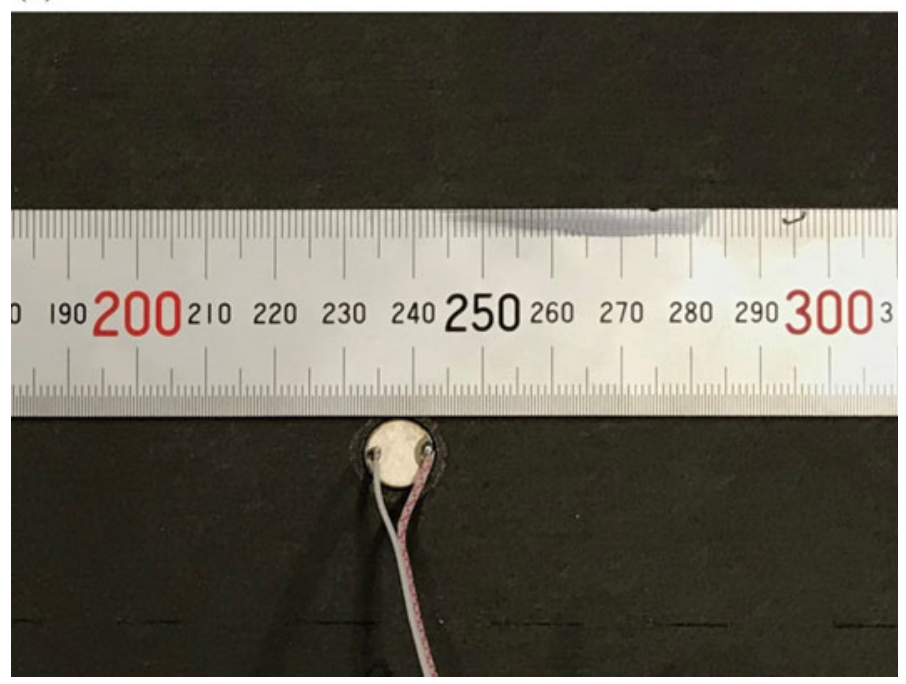

Fig. 4.1 Photographs of the experimental setup for the electromechanical impedance (EMI) measurements; a impedance analyzer and $\mathbf{b}$ surface-bonded piezoelectric sensor

Considering the difference in the root mean square (RMS) values, it can be noted that in this frequency region, an increase in the energy (RMS) value is observed because all the differential values are non-negative (Fig. 4.2). Next, the location of the resonance peak in the considered bandwidth was tracked for all samples. In most of the cases, the peak shifted left. The relative frequency shift between the two reference samples was only $-0.027 \%$ (Fig. 4.3). Considering the absolute values, all the results 


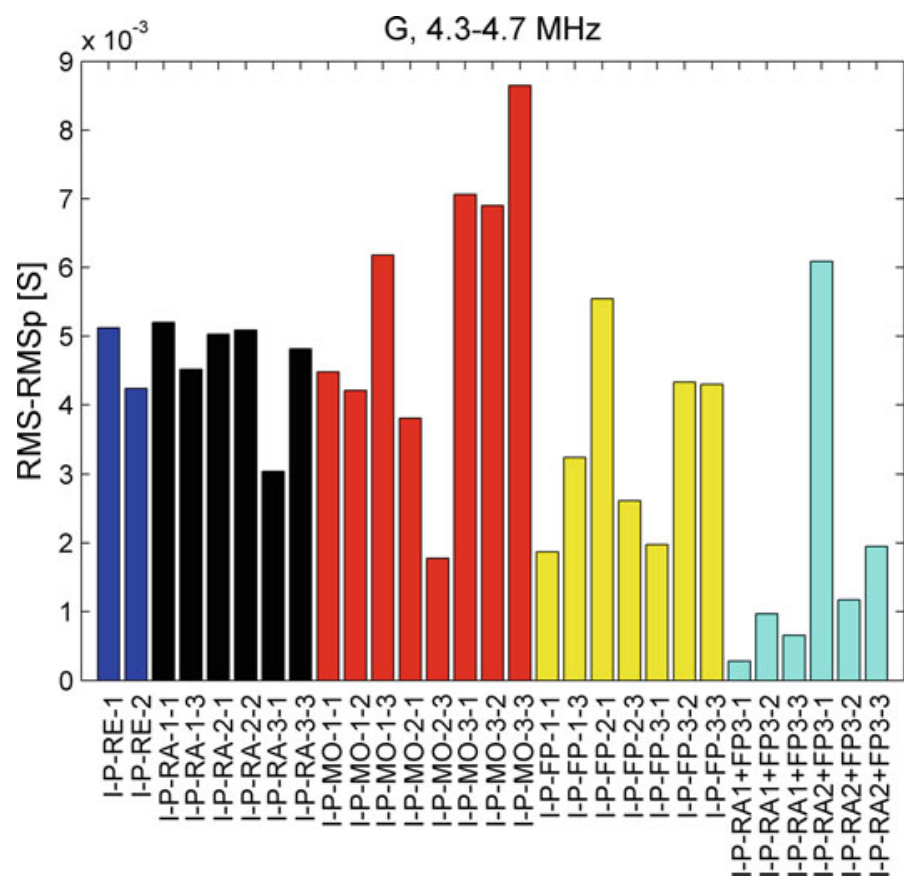

Fig. 4.2 Differences of the RMS values of EMI sensors bonded to flat coupon samples and free sensors for the production scenario

for the contaminated samples lie above this threshold. In general, the frequency shift increases with the increase of the moisture and fingerprint contamination level. However, there are some exceptions, such as very low values for the I-P-MO-1-2, I-P-FP-1-3, and I-P-RA2+FP3-3 samples and a high value for the I-P-FP-1-1 sample.

The sample set investigated for the repair scenario comprised three reference samples, nine thermally degraded samples, nine samples contaminated with de-icer, nine samples modified by a faulty curing of the adhesive, and six samples with a combined contamination of thermal degradation and contamination with de-icer. After considering the registered spectra, some of the results were rejected (I-R-RE-2, I-R-TD-1-2, I-R-TD-2-2, I-R-TD-3-3, I-R-FC-3-2, I-R-TD1+DI2-1, I-RTD1+DI22) due to the different shapes of the spectra. Moreover, previous results have shown that the I-R-TD-3-1 sample was delaminated [8]. The spectra were inspected in the 4.25-4.70 MHz region. A local resonance was observed, except for the sample with delamination. The presence of this local resonance is indicated by the increase in RMS values with respect to the free piezoelectric sensors (Fig. 4.4). The resonance location was tracked with respect to the I-R-RE-1 sample result. The relative frequency shift is shown in Fig. 4.5, and most of the samples were characterized by leftward peak shift. Considering the absolute value, all the samples are above the reference threshold defined by the I-R-RE-3 result, with the exception of the I-R-TD-2-3 sample. In the case of the thermal treatment (TD), the increase of the treatment temperature 


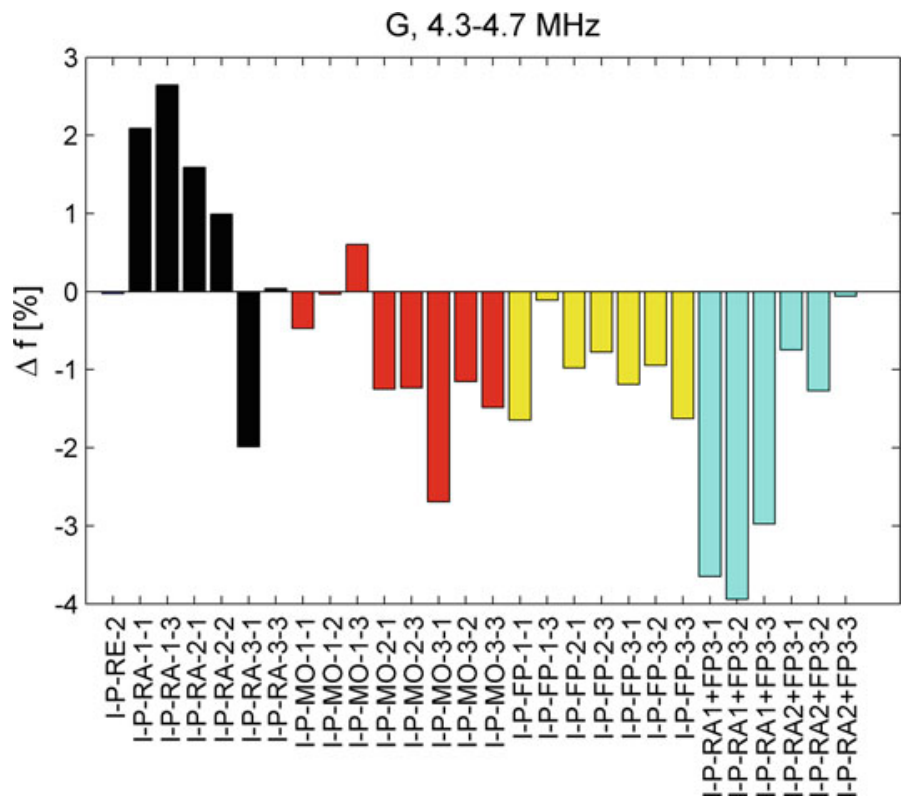

Fig. 4.3 Tracking of local EMI resonance in the 4.3-4.7 MHz range; frequency shift calculated in relation to the I-P-RE-1 sample

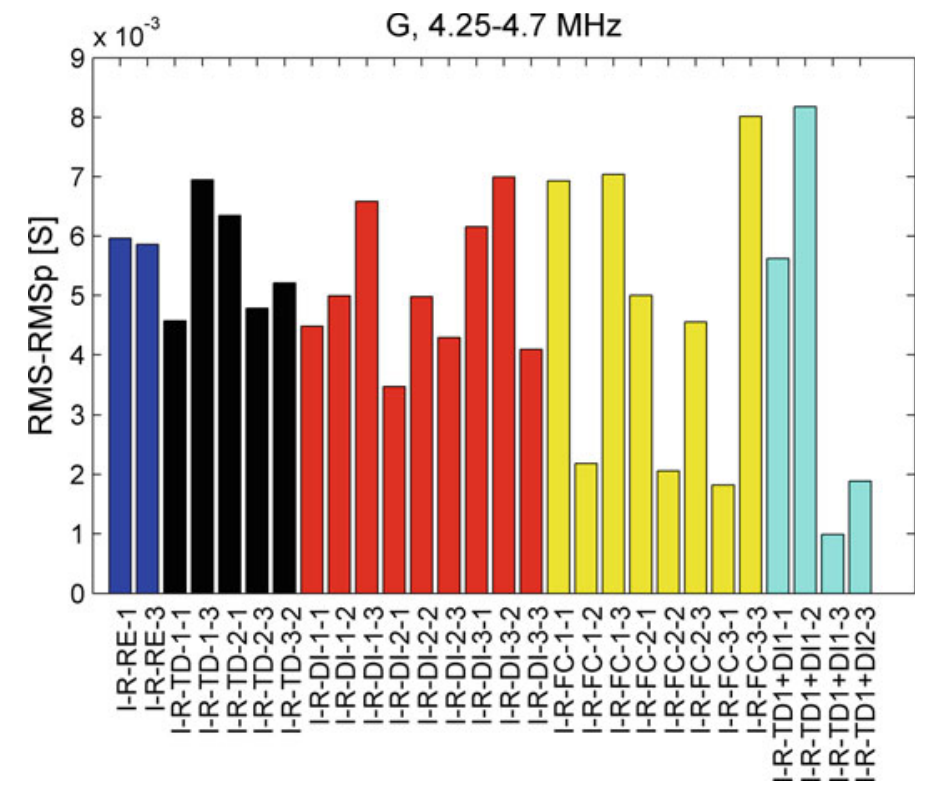

Fig. 4.4 Differences of the RMS values of EMI sensors bonded to flat coupon samples and free sensors for the repair scenario 


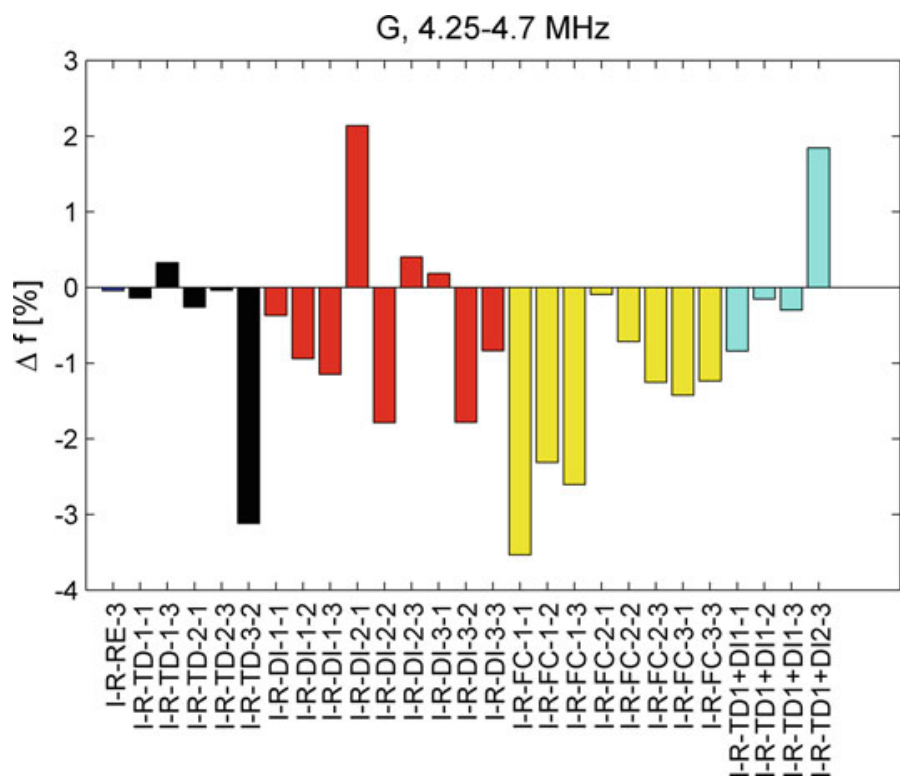

Fig. 4.5 EMI conductance peak shift in the 4.25-4.70 MHz range for the flat coupon samples (repair scenario)

results in an increase of the shift. In the case of the de-icer (DI) contamination, such dependence was not observed. The most intensive faulty curing was applied to the I-R-FC-1-x samples, and this was also observed for the results obtained with the EMI method. The results presented in Fig. 4.5 also show a sensitivity of the frequency shift to the mixed contamination level. However, these results should be treated with caution because only one sample (I-R-TD1+DI2-3) at the higher level of contamination was able to be considered.

\subsubsection{EMI Results on Pilot Level Samples}

In the case of the production scenario, the pilot samples were curved. The inspected conductance spectra were different, which should be attributed to the different sample geometry. No clear appearance of additional resonances was observed. Therefore, the highest peak in the spectrum was tracked. The frequency shift calculations were performed in relation to the II-P-RE-1 sample (Fig. 4.6). Four samples were characterized by a rightward shift, while three showed a leftward shift. Considering the absolute values of the shifts, the difference between the reference samples is $1.71 \%$, so only three contaminated samples out of the six were above this threshold. The sensitivity to the contamination level was not clear in the considered case.

The pilot samples from the repair scenario had a scarfed bonding area. The spectra of free sensors and the spectra after bonding these sensors to the scarfed samples 


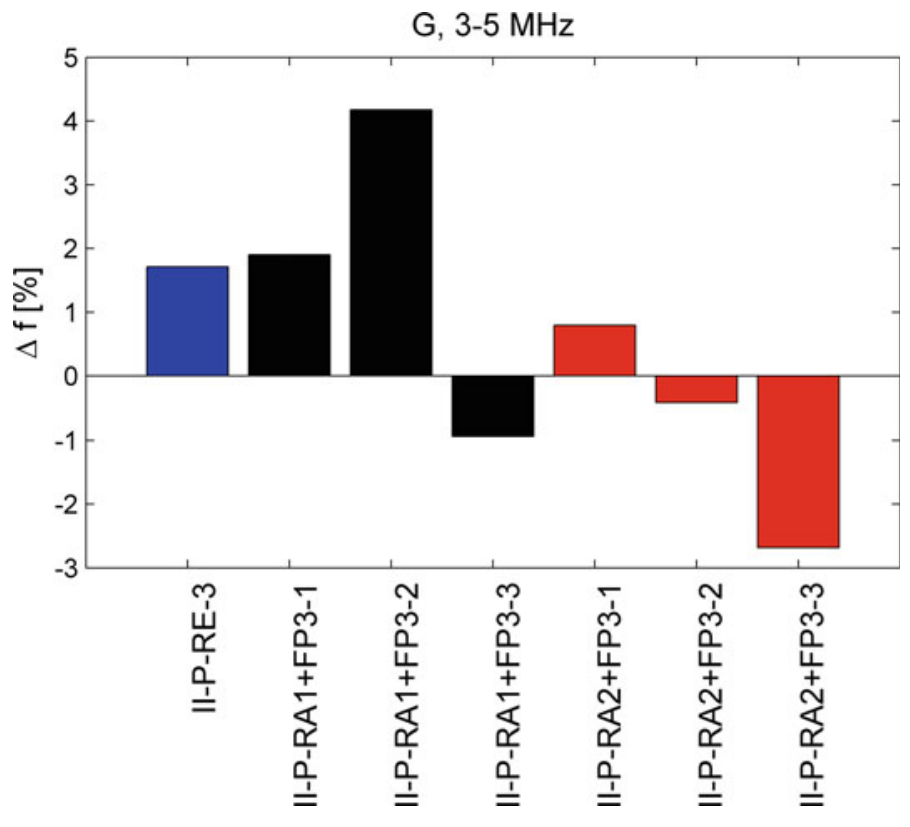

Fig. 4.6 EMI conductance local maximum shift in the range of 3-5 MHz for the curved samples

were inspected. The sample II-R-TD1+DI2-2 was rejected from the analysis due to the different shapes of the spectrum. For the rest of the samples, a local resonance was observed in the conductance range 3.95-4.15 MHz. In this frequency range, the root means square values were calculated for both the free sensors (RMSp) and the samples with bonded sensors (RMS). It was observed that all the differences (RMS-RMSp) are non-negative (Fig. 4.7). This indicates the influence of the sensor bonding to the inspected samples. This frequency region was further inspected, and the frequency shift was tracked in relation to the II-R-RE-1 sample. It can be observed that there were significant differences between the reference samples (Fig. 4.8a), which were higher than for the samples with contaminated adhesive bonds. The changes in the magnitude of this resonance in relation to the same reference sample are presented in Fig. 4.8b. The magnitude for most of the samples increased, while in two cases it dropped. In comparison with the reference samples, it can be noted that the absolute magnitude is higher for all the samples with contaminated bonds, except for the II-R-TD1+DI1-2 sample. While differences were detected, it cannot be stated that the contamination levels can be distinguished. 


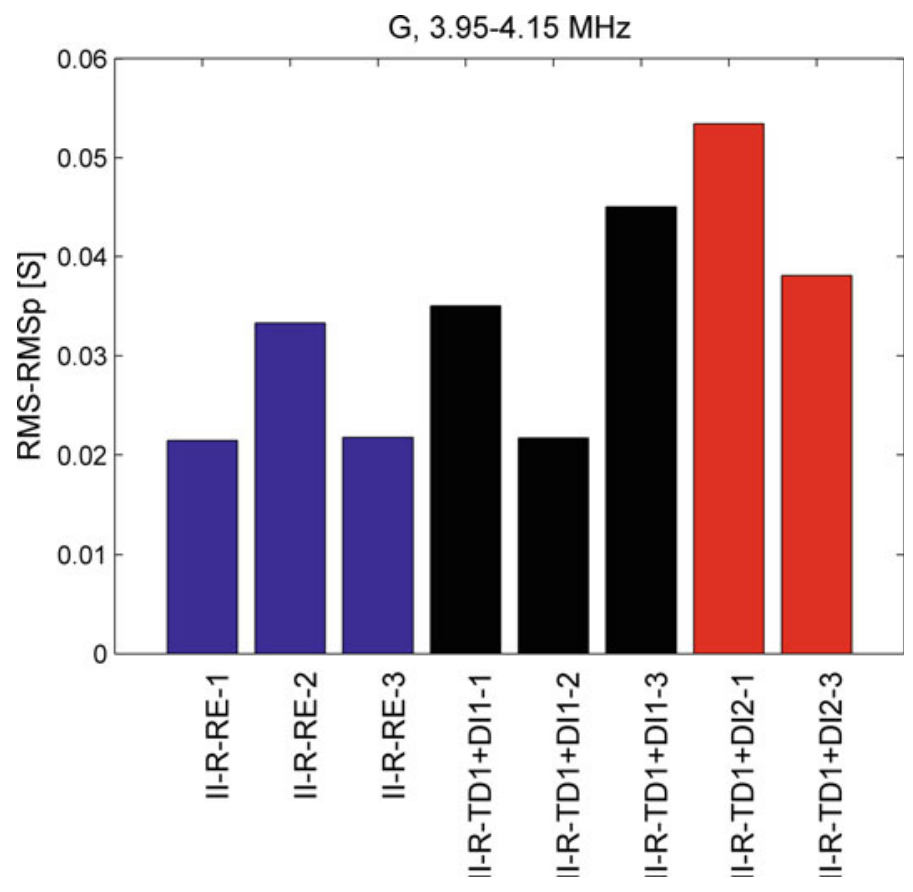

Fig. 4.7 Differences of RMS values of sensors bonded to scarfed samples and free sensors

\subsection{Laser Shocks}

In this section, we describe the principle and instrumentation of the Laser Shock Adhesion Test (LASAT) method and detail the results recently obtained in the ComBoNDT project.

\subsubsection{Principle and Instrumentation}

LASAT relies on the recombination of shock waves produced within the tested material to generate localized tensile stress. To produce the required shock, a high-power $\mathrm{Nd}-\mathrm{YAG}$ laser is used. Its energy often varies between 1 and $40 \mathrm{~J}$, and short pulses (for 3-20 ns) are necessary to generate the required power density (from 1 to 10 $\mathrm{GW} / \mathrm{cm}^{2}$ ). When the laser reaches the sample surface, a thin layer of the material is vaporized in dense plasma (within the pressure range of 1-5 GPa). Upon expanding, this plasma creates shock waves within the material Fig. 4.9. These shock waves (full lines in Fig. 4.10), followed later by release waves (dashed lines in Fig. 4.10), travel to the back face of the sample. Once the shock waves reach the back face, they 


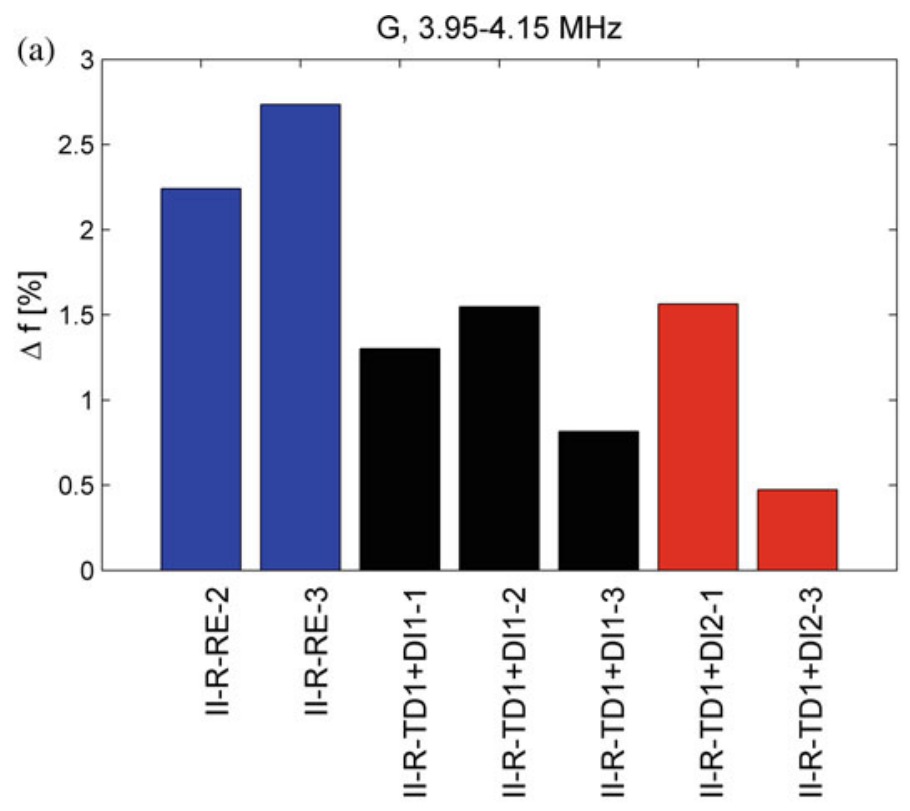

(b) $\quad$ G, $3.95-4.15 \mathrm{MHz}$

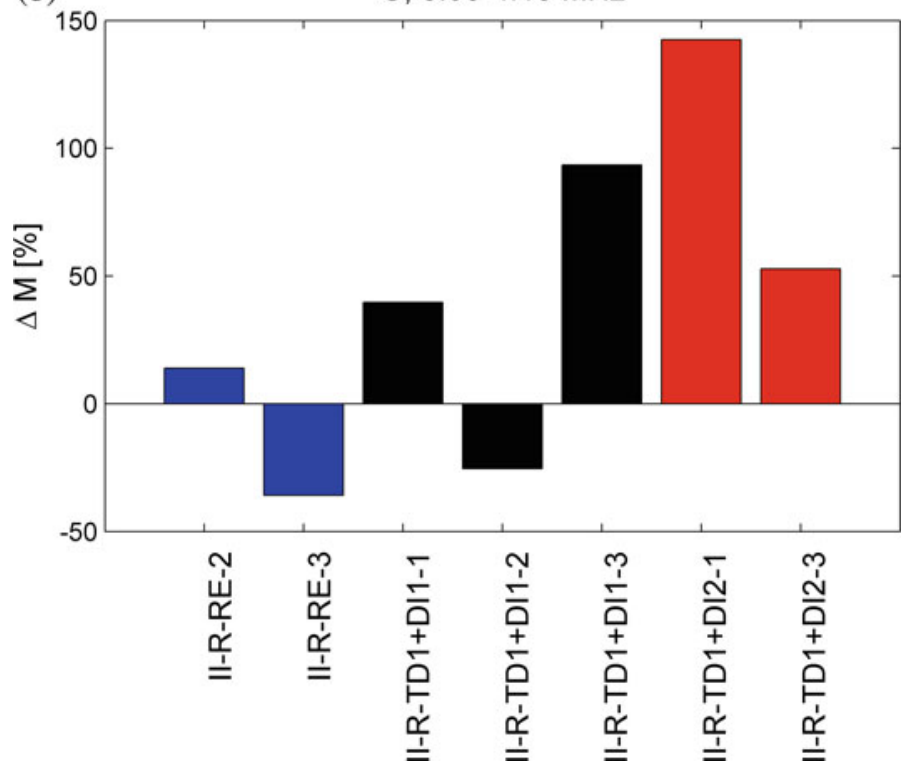

Fig. 4.8 a Conductance local maximum shift in the range of 3.95-4.15 MHz for the scarfed samples; the relative shift was calculated in relation to the II-R-RE-1 reference sample; $\mathbf{b}$ change of the conductance magnitude in the range of $3.95-4.15 \mathrm{MHz}$ for the scarfed samples; the relative change was calculated in relation to the II-R-RE-1 reference sample 


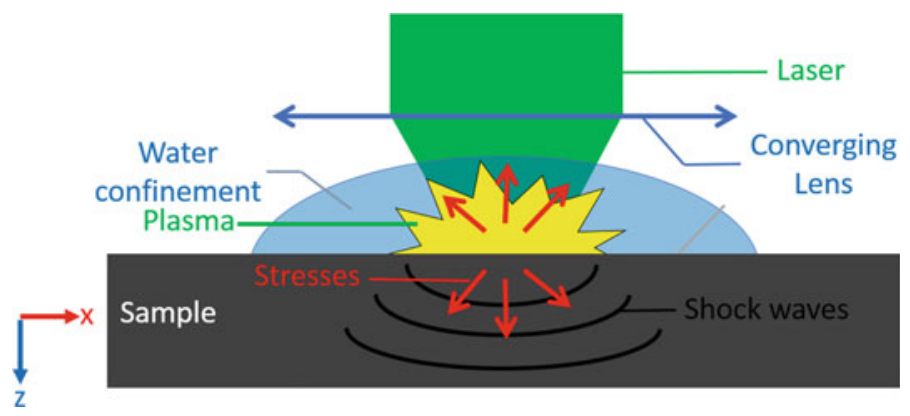

Fig. 4.9 Generation of shockwaves using a laser

Fig. 4.10 Time/space diagram describing the shock wave behaviour within a material

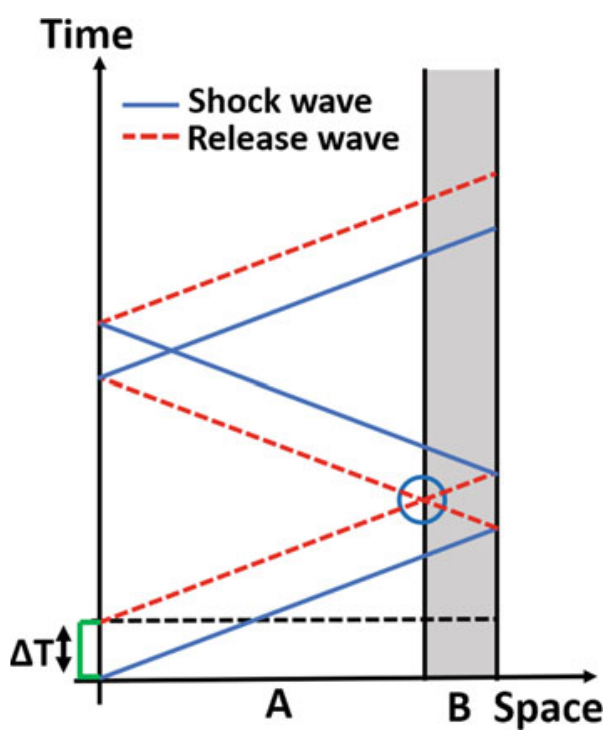

are reflected as release waves. When the latter intersects the incident release wave, tensile stress is created (circle in Fig. 4.10).

Studies have shown that it is possible to increase the generated stress by using the water-as a confinement medium [9]. Hence, stresses can be multiplied by up to four times for the same laser intensity, allowing less powerful and more compact lasers to be used for this application.

It is possible to link the laser intensity applied to the surface to the pressure generated within the material [10]

$$
\mathrm{P}(\mathrm{GPa})=0.01 \sqrt{\frac{\alpha}{\alpha+3}} \sqrt{\mathrm{I}\left(\mathrm{GW} / \mathrm{cm}^{2}\right.} \sqrt{\mathrm{Z}_{\mathrm{rel}}\left(\mathrm{kg} \mathrm{m}^{-2} \mathrm{~s}^{-1}\right)}
$$

where 
- $\alpha=$ part of the energy being used for the gas ionization

- $\mathrm{I}=$ laser intensity

- $\mathrm{Z}=$ the relative impedance

The relative impedance is a ratio between the material impedance of the sample $\left(Z_{\text {sample }}\right)$ and the confinement impedance $\left(Z_{\text {conf }}\right)$

$$
Z_{\text {rel }}=2 \times \frac{Z_{\text {conf }} \cdot Z_{\text {sample }}}{Z_{\text {conf }}+Z_{\text {sample }}}
$$

Shock generation using a laser was first introduced by Vossen [11] to test the adherence of thin layers. Following these initial studies, Yuan [12] dealt in depth with the measurement of the interfacial stresses generated by this technique. More recently, Boustie [13] and Bolis [14], extended the laser shock technique to thicker metallic coatings. Gilath was among the first to test bond adhesion using laser shock generated tensile stresses [15]. Later, composite materials [16] and bonded composite materials [17] were also studied using the Laser Shock Adhesion Test.

Beginning in 2010, the ENCOMB project aimed at the development of new Non-Destructive Tests (NDT) that are capable of assessing the strength of bonded composite structures, and LASAT showed great promise in the detection of weak bonds and had the advantage of being contact-free. However, there was a major drawback: the rear part of the composite structure can be damaged within the range of laser intensity used to test the bond strength.

Based on the results of the project, three new configurations were proposed to control the tensile stresses inside the material by adjusting a temporal parameter of the laser:

- Variable laser pulse duration [18] (Fig. 4.11a);

- Double front face shocks [19] (Fig. 4.11b);

- Symmetrical laser shocks (Fig. 4.11c).
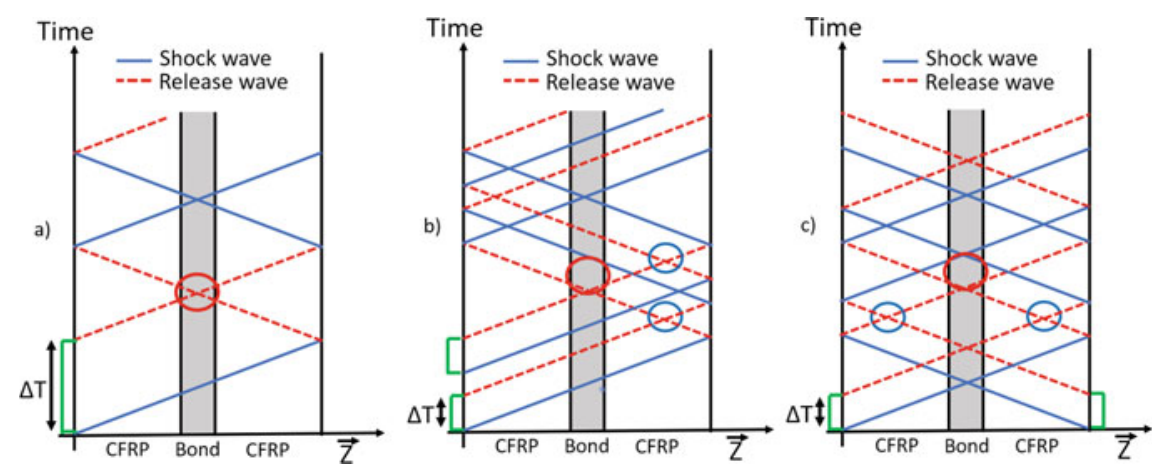

Fig. 4.11 LASAT optimisations: a variable laser pulse; $\mathbf{b}$ double shock; and $\mathbf{c}$ symmetrical shot 

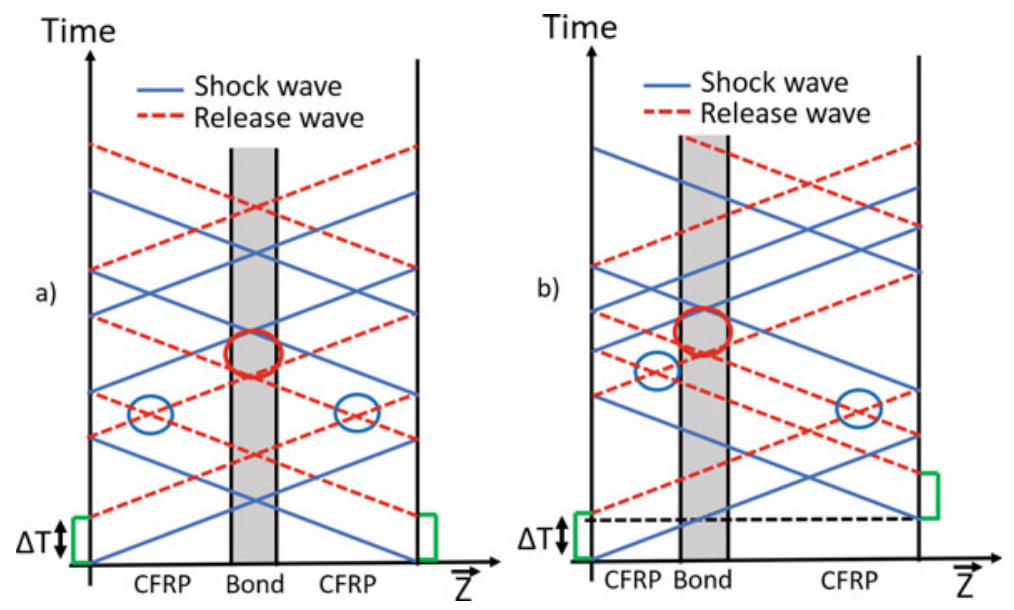

Fig. 4.12 Symmetrical LASAT: a without time delay and $\mathbf{b}$ with time delay

The symmetrical laser shock was implemented for ComBoNDT. The secondary tensile stress area, which is similar to that found in the single shot setting, is still noticeable (blue circles in Fig. 4.11c). However, in the symmetrical configuration, an even higher tensile stress area is located where both reflected shock waves cross (red circle in Fig. 4.11c). The position of this stress depends only on the time delay between both shots (Fig. 4.12).

For a given location $\boldsymbol{A}$, the time delay can be calculated using the following equation:

$$
\Delta \mathrm{T}=\sum_{\mathrm{i}} \mathrm{t}_{\mathrm{li}} \times \frac{\rho_{\mathrm{li}}}{\mathrm{Z}_{\mathrm{li}}}-\sum_{\mathrm{j}} \mathrm{t}_{\mathrm{rj}} \times \frac{\rho_{\mathrm{rj}}}{\mathrm{Z}_{\mathrm{rj}}}
$$

where $\mathrm{t}_{1 \mathrm{i}}$ represents the thickness of a layer situated to the left of $\boldsymbol{A}, \rho_{\mathrm{l}_{\mathrm{i}}}$ is its density, and $\mathrm{Z}_{\mathrm{li}}$ is its impedance. In the same way, each variable with the index letter " $\mathrm{r}$ " refers to the right part of $\boldsymbol{A}$.

\subsubsection{Instrumentation and Method}

Figure 4.13 describes the different steps of a LASAT procedure. It starts with the characterization in situ of the bond and the material properties of a reference sample. This initial step serves two purposes, namely finding the bond threshold and gathering the material data. Using these data, a series of numerical simulations are performed to optimize the laser parameters of intensity, time delay, and focal spot size. The sample is then prepared with an aluminium tape applied to both areas where the laser shot will occur. The tape serves as a sacrificial layer, preventing the sample from being ablated by the laser. It also ensures good repeatability of the shots. Indeed, 


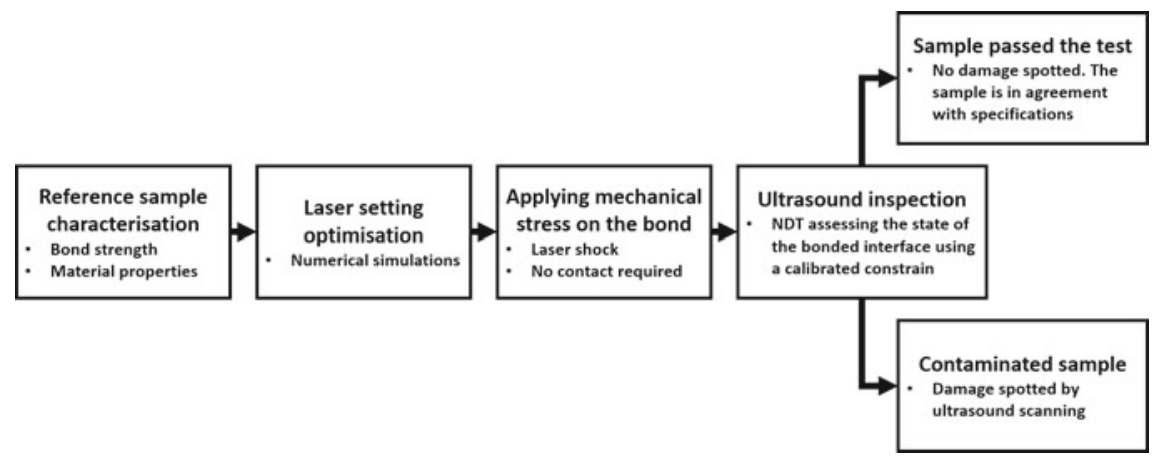

Fig. 4.13 Laser shock adhesion test methodology

the generated plasma significantly depends on the laser/matter interaction. By only using aluminium as a protective sacrificial layer, all parameters are fully controlled during the laser shot process.

The laser intensity is set to a percentage of the bond threshold found during the first step. In the aeronautical industry, a bond is considered faulty or weak if its adherence is below $80 \%$ of the nominal adherence defined in the specifications.

Once all the parameters are set, the laser shot occurs. If the bond is weak, the high tensile stress generated on the bond will create a small gap at the interface between the composite and the joint. This can be detected using ultrasound scanning, a reliable method that is often used to reveal these kinds of defects.

If no damage is spotted, then the sample is considered sound and has passed the test. Otherwise, the bond shows weakness and does not follow the specifications, meaning the component cannot be used and has to be replaced. Thus, this technology is only destructive when the tested part is not in accordance with the specifications. If the part is sound, it can be used as it is.

\subsubsection{Description}

LASAT is currently performed on the Hephaistos platform (Fig. 4.14). This facility consists of a high-power GAÏA laser (Fig. 4.14a), which has the particularity of consisting of two different lasers, with the beams being polarized from one another by $90^{\circ}$. Three different platforms are currently powered by the laser: one for laser shock peening studies, one for single shot LASAT, and one for symmetrical LASAT. The final one (Fig. 4.14b) uses a polarizer that, due to the two different polarisations, splits the main beam into two different laser beams (beam A and beam B). Using mirrors and lenses, each ray is then carried to the prepared sample (Fig. 4.14c).

In order to ensure a good control over the pressure spatial repartition, Diffractive Optic Elements (DOE) are added to both beam paths. These optics smooth the focal spot, creating a homogeneous focal spot on the sample (Fig. 4.15). 


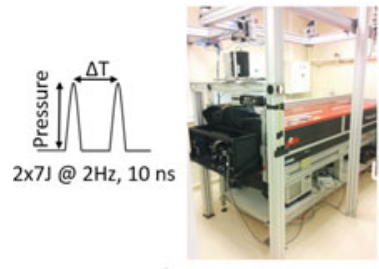

a)

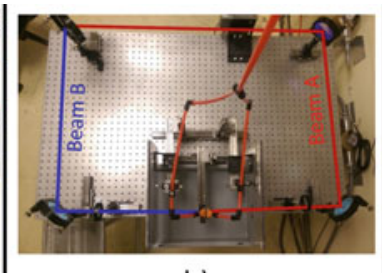

b)

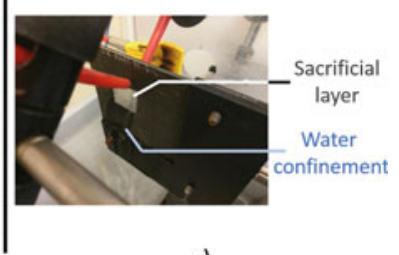

c)

Fig. 4.14 Hephaistos facility: a GAÏA laser from Thales; b symmetrical experimental setup; c sample setup with water confinement and the protective aluminium tape
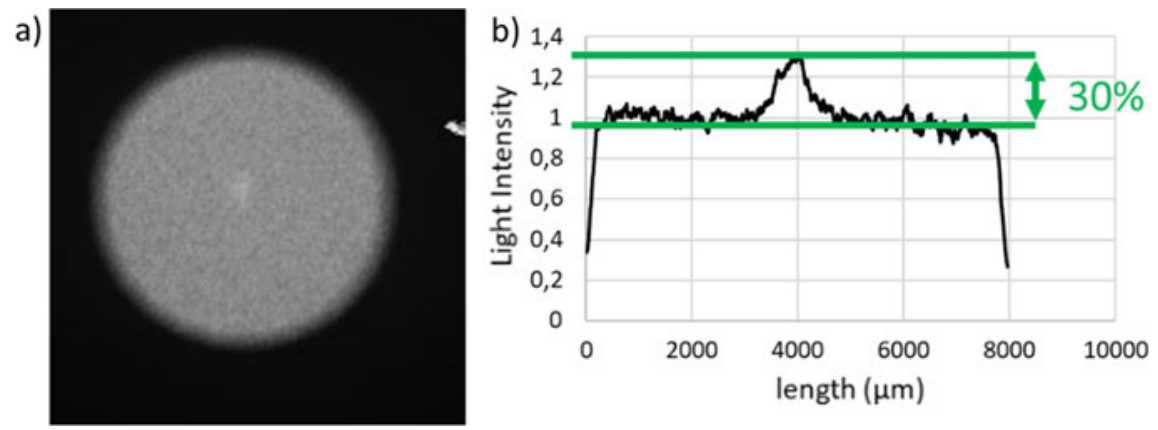

Fig. 4.15 DOE: a focal spot exiting a DOE and $\mathbf{b}$ light repartition over the focal spot

A series of 20 shots were realized for 11 different laser intensities, ranging from 5 to $100 \%$ of the maximum intensity. The standard deviation for each intensity level was less than $1 \%$, meaning that each shot is reproducible and can be used for comparison purposes.

As specified earlier, ultrasound scanning is required to examine the structure after the laser shock. These are calibrated while the reference sample is being studied in situ to obtain the material data. In the case of composite layer bonding, ultrasound is needed to find the signature of the front face (blue in Fig. 4.16), the bond (yellow in Fig. 4.16), and the back echo (green in Fig. 4.16).

Before shooting the sample, a simulation must be run to optimize the laser parameters, whereby the position and width of the maximum tensile stress are analyzed and modified if necessary (Fig. 4.17). The challenge of such numerical calculations resides in the simulation of the high strain rates generated by the technology $\left(\sim 10^{7} \mathrm{~s}^{-1}\right)$. Because the material used is orthotropic, it is not possible to describe the state of the matter using an equation of state, as is usually done with isotropic materials. The simulation of damage also requires more advanced laws to take into account the different damaging modes of composite materials. 
a)

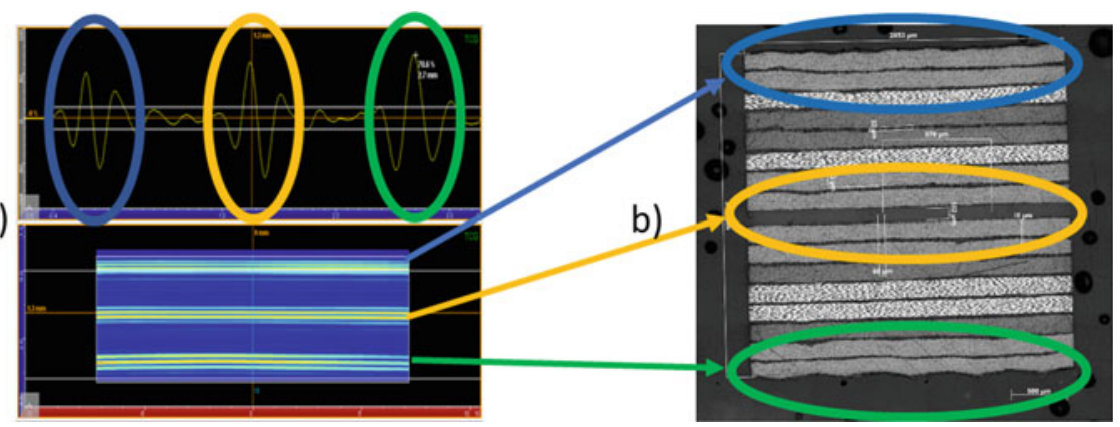

Fig. 4.16 In situ studies: a reference ultrasound of the benchmark structure and $\mathbf{b}$ photomicrograph of the benchmark structure
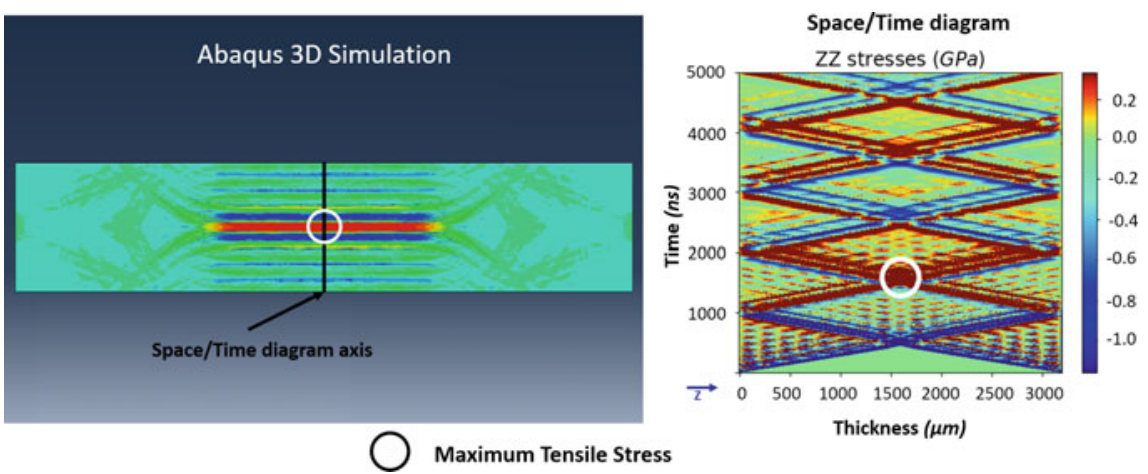

Fig. 4.17 Finite element simulation of a laser shock

\subsubsection{Laser Shock Results}

\subsubsection{Laser Shock Results on Coupon Level Samples}

Prior to the large-scale experiments, an initial study was performed to assess whether ultrasound scanning is efficient enough to spot a defect created with a laser shock. Each coupon was divided into 16 different areas, whereby the laser intensity (ranging from 5 to $95 \%$ ) was raised from one area to the other. The first area to be opened defined the sample's bond threshold (red circle in Fig. 4.18a). This was followed by a study in which both the first opened area and the one preceding it (red circle in Fig. 4.18b) were cold mounted and examined using optical microscopy.

In each case, the initial default was located on the bond, and none of the composite laminate was damaged. This study also showed that a defect detected using ultrasound scanning (Fig. 4.18c) can be confirmed by a direct inspection through photomicrographs (Fig. 4.18b). These results lead to the conclusion that ultrasound scanning is a reliable technique to reveal defects generated using laser shocks. 

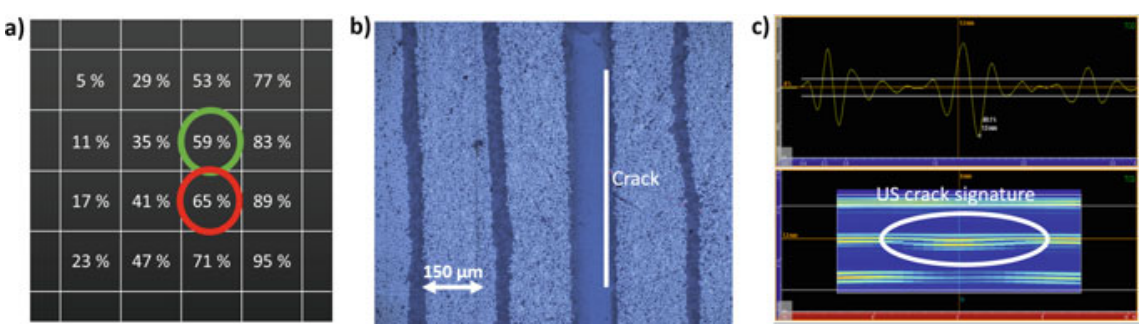

Fig. 4.18 a Shot layout over a coupon sample; $\mathbf{b}$ photomicrograph of an open bond; $\mathbf{c}$ related B-scan

Several procedures can be used to assess bond strength using LASAT. In this study, one specific area was subjected to incremental laser intensity. After each shot, an ultrasound scan was performed to verify whether or not the bond had failed at this given intensity. If it broke, then that energy was assumed to be the bond threshold for that sample. On the other hand, if it remained intact, the energy was increased. Samples representative of both the manufacturing and the repair scenarios were tested. In each case, the reference sample sets the standard bond threshold. When testing a contaminated sample, if the threshold found was to be lower than the standard one, the LASAT was considered successful. The results for the manufacturing samples are summarized in Fig. 4.19.

Each bar represents the energy required to open the bond. The reference sample is presented in blue, while orange is the sample from the release agent contamination, green represents the moisture contamination, and yellow is for the fingerprint contamination. The red line represents the scanning intensity. If these samples were from an actual component, then this intensity should be chosen to assess whether or not a bond is weak. Every sample that fail before this point should be considered weak. The LASAT successfully differentiated eight out of nine contaminated samples. The only

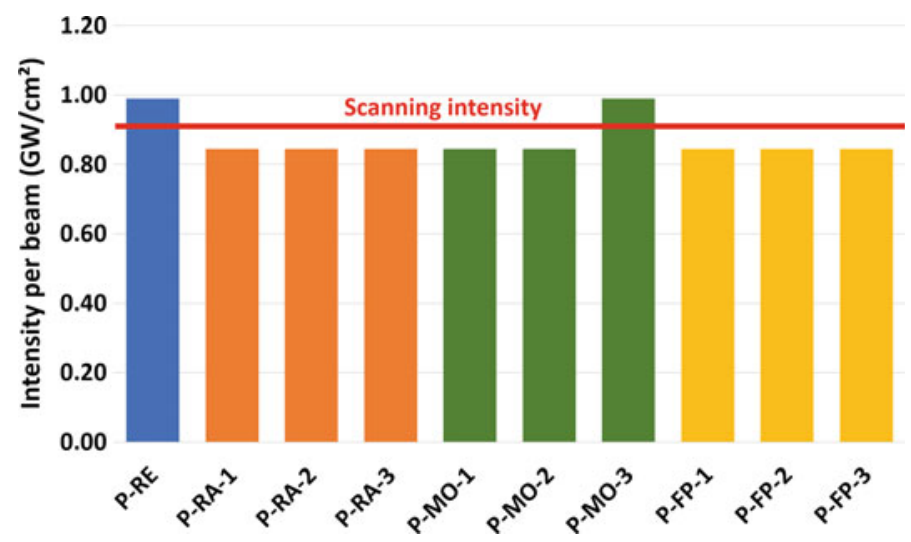

Fig. 4.19 Summary of results for the manufacturing samples 


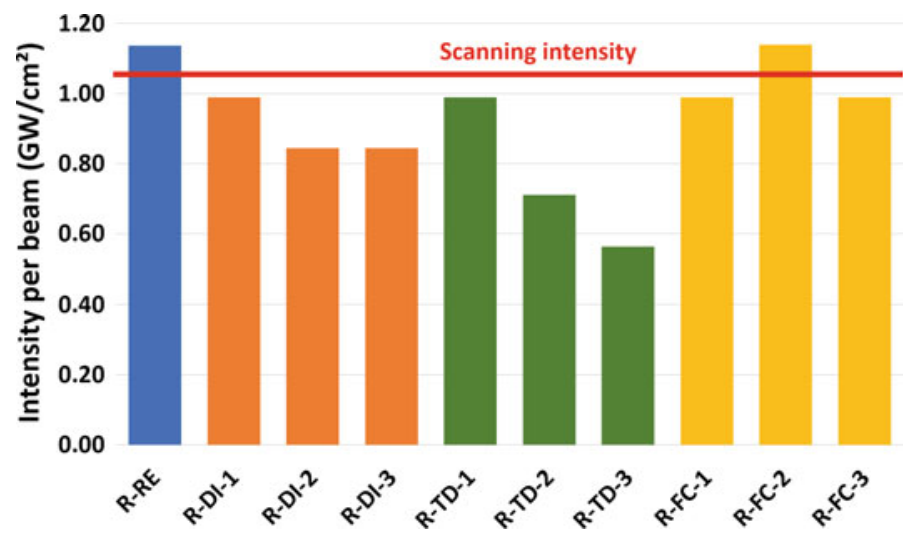

Fig. 4.20 Summary of the results for the repair samples

sample with the same intensity as the reference sample was the one with the highest moisture contamination. It has already been observed that following an increase in water intake, an epoxy-based adhesive bond can have a higher mechanical strength than a non-contaminated bond [20]. Since the bond was mechanically tested, this result is in accordance with the literature. The repair scenario samples also showed good results for the LASAT (Fig. 4.20). The reference sample is again represented in blue, while the results for the de-icer are in orange, those for the thermal degradation in green, and the samples for the false curing are in yellow. For the first two contamination types, the different levels of contamination could be detected, especially for the thermally degraded sample, whereby each level of contamination has its own threshold. The results are not as clear for the faulty curing. This type of contamination is not easy to control, and these samples had already shown delamination before any test was performed on them. Even though two out of three samples were shown to be contaminated, this is hard to state conclusively given the initial state of the tested samples.

It has also been observed that the repair reference sample and the production reference sample had different bond thresholds, namely $0.99 \mathrm{GW} / \mathrm{cm}^{2}$ for the production scenario and $1.15 \mathrm{GW} / \mathrm{cm}^{2}$ for the repair scenario. Photomicrographs taken from both bonds revealed a different crack pattern Fig. 4.21.

As the repair sample was ground down to the fibre before the epoxy-based adhesive was applied, the cracks tended to propagate within the bond. Meanwhile, for the production sample, a thin layer of prepreg matrix was still present, and it was noticed that the crack mainly propagated along the interface between the bond and the prepreg matrix. Studies have already shown that the interface strength can be higher than the actual mechanical strength of the glue [21]. One of the explanations for this behaviour was the difference in surface tension between the prepreg matrix and the actual composite fibre, which may have led to a different bond quality with the FM300 used for the joint. 

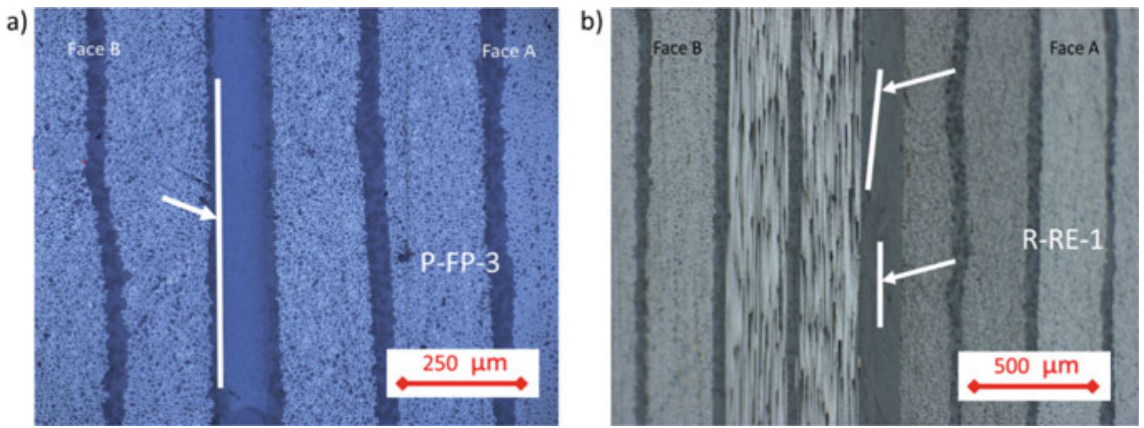

Fig. 4.21 Photomicrographs: a production sample and $\mathbf{b}$ repair sample

To conclude, the LASAT gave good results for the coupon samples, and these were in accordance with the results found during the $G_{I C}$ and $G_{I I C}$ tests [22]. In certain cases, the test was also able to identify the different levels of contamination.

\subsubsection{Laser Shock Results for the Pilot Level Samples}

Three different scenarios were tested for the pilot samples: multiple contaminations, scarf bonding, and curved samples.

The results are mixed for coupon samples with multiple contaminations (Fig. 4.22). The LASAT did not differentiate production samples with multiple contaminations from the reference sample, sample, and the intensity required to open the faulty joints was the same as for the standard. The results are also hard to explain in the repair scenario. The technology detected the least contaminated sample but was unable to clearly detect the most contaminated sample. However, the same contaminations on the scarfed samples showed much better results (Fig. 4.23). In this case, the LASAT technology was effective at differentiating a sound bond from a weak one. The difference between the results for the coupon samples and the scarfed samples is not yet fully understood; the bond geometry may have played a
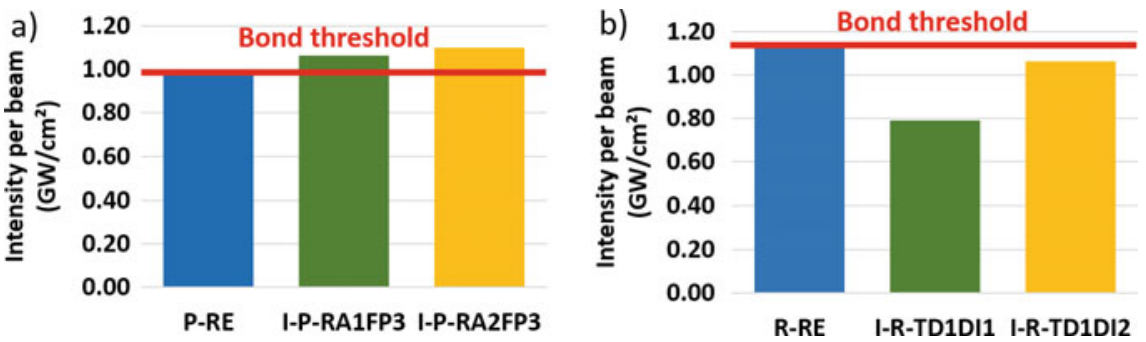

Fig. 4.22 Summary of the results of multiple contaminations on coupon samples for a production samples and $\mathbf{b}$ repair samples 


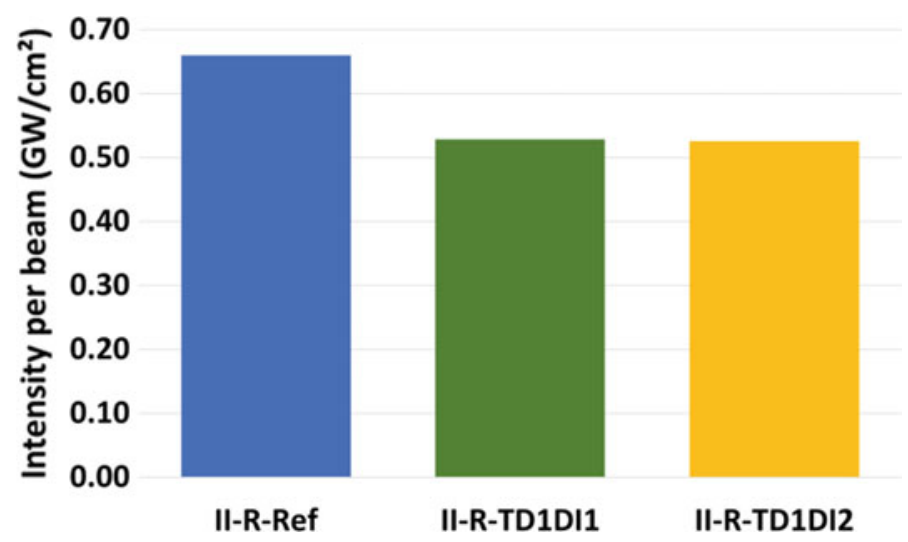

Fig. 4.23 Summary of the results for multiple contaminations on scarfed samples

part in the crack detection/propagation within the contaminated samples. This technology also heavily relies on a standard sample, and the reference used for the coupon samples may have been produced with different parameters. Furthermore, the curved samples were highly porous and did not follow the specifications. Therefore, no relevant conclusion can be derived from the results and further study is required in order to draw decisive conclusions for these samples.

\subsection{Nonlinear Ultrasonic Technique}

In this section, we describe the principle and instrumentation of the nonlinear ultrasonic technique (NUS) and detail the results recently obtained in the ComBoNDT project.

\subsubsection{Principle and Instrumentation}

Conventional ultrasonic NDT instruments used in industry and technology make use of the so-called linear elastic response of materials, which results in amplitude and phase variations of the input signal due to its interaction with any defects present. The nonlinear approach to ultrasonic testing involves the nonlinear material response related to the frequency changes of the input signal. These spectral changes are caused by the nonlinear dynamics of solids, which range in scale from the inter-atomic level for perfect materials to meso- and macro-scale nonlinearity for damaged areas [23-25]. In many cases, monitoring material nonlinearity directly reveals vulnerable areas within a material or product with sensitivity far superior to traditional ultrasonic inspection methods [26]. Numerous studies have confirmed 
that the increase in nonlinearity is closely related to a "softening" of the material due to, e.g., fatigue, cracking, and internal interfaces. This also correlates with the general characteristic of acoustic nonlinearity that is related to the thermal expansion of the material, which is negligible for stiff materials and increases significantly for soft solids [27].

A conventional method to evaluate nonlinearity in composite coupons is based on the second harmonic measurements for propagating plate (Lamb) modes [28, 29]. The approach proposed in this research makes use of a local nonlinear response of the adhesive bond. A contaminated bonding layer with weaker adhesion is supposed to have a higher nonlinearity that enables the difference in bonding quality to be recognized, evaluated, and visualized.

The nonlinear technique is based on the local generation of high amplitude vibrations and the detection of the higher harmonics in the excitation area. Commercial piezo-actuators (isi-sys $\mathrm{GmbH}$, Germany) with a frequency response extending from the low $\mathrm{kHz}$ into the high $\mathrm{kHz}$ range (above $100 \mathrm{kHz}$ ) were used in the experiments. The actuators were driven by a CW voltage generated by an HP 33120A arbitrary waveform generator and were vacuum-attached to one of the sides of the sample, while nonlinear vibrations were measured on the opposite side. The generator was combined with a HVA-B100 amplifier to result in a 10-40 V input amplitude for the piezo-actuator. To measure and analyze the frequency content of the vibrations generated locally in the excitation area, a scanning laser vibrometer (SLV, Polytec 300) operating in the vibration velocity mode with a maximum frequency bandwidth of $1.5 \mathrm{MHz}$ was used.

The dynamic range of the SLV measurements (100-120 dB) lies well beyond the level of nonlinear frequency components. In the experiments, the vibration amplitude was measured as being in the range of $(4-5) \times 10^{-8} \mathrm{~m}$, thus the local strain that developed directly in the excitation area was approx. $10^{-5}$. This strain is sufficient for the manifestation of noticeable local nonlinearity in composite materials.

To avoid an impact of reflections on the local vibration in the excitation area, the edges of the samples were covered with a dissipative material and a high vibration frequency was chosen $(49 \mathrm{kHz})$. With these precautions, the spectra and temporal patterns of the vibrations were measured directly in the acoustic source area (a circle with approx. $5 \mathrm{~mm}$ radius), whereby plate wave propagation was not yet involved.

Each value for the fundamental frequency vibration velocity $\left(\mathrm{v}_{0}\right)$ and the higher harmonic components $\left(\mathrm{v}_{\mathrm{n}}\right)$ measured in the probing area was used for the evaluation of the nonlinear ratio

$$
\mathrm{N}_{\mathrm{i}}=\sum_{\mathrm{n}} \frac{\mathrm{v}_{\mathrm{n}}^{2}}{\mathrm{v}_{0}^{2}}
$$

An average value of the nonlinear ratio in the probing area was then calculated with 


$$
\mathrm{N}=\sum_{\mathrm{i}=1}^{\mathrm{m}} \frac{\mathrm{N}_{\mathrm{i}}}{\mathrm{m}}
$$

where $\mathrm{m}$ is the number of measurements; the standard deviation of the results was estimated

$$
\Delta N=\sqrt{\sum_{i=1}^{m} \frac{\left(N_{i}-N\right)^{2}}{m(m-1)}}
$$

The measurements were repeated at various points over the central part of the sample to provide the relative error $\Delta \mathrm{N} / \mathrm{N} \leq 10-20 \%$.

The nonlinear ratio $N_{i}$ is part of the vibration energy $\left(\sim \mathrm{v}_{0}^{2}\right)$ converted into the higher harmonics $\left(\sim v_{n}^{2}\right)$ so that it clearly quantifies material nonlinearity. To avoid the influence of the amplitude-dependent effects in the estimation of the nonlinear ratio $\mathrm{N}$, the amplitude of the input voltage of the transducer was kept constant $(20 \mathrm{~V})$ over the course of the measurements. As a result, in all the samples measured the fundamental vibration amplitude was virtually constant within an approx. $10 \%$ deviation.

\subsubsection{Nonlinear Ultrasonic Technique Results}

\subsubsection{Nonlinear Ultrasonic Technique Results for Coupon Level Samples}

For the production scenario, the following kinds of contaminants were studied: release agent (RA), fingerprint (FP), and moisture (MO). The repair scenario investigations comprised a de-icing fluid (DI) contamination, thermal degradation (TD), and faulty curing (FC). For each type of contamination in both scenarios, a set of three samples for every level of contamination was prepared to provide reliable statistics. The results of the measurements and calculations of $N$ for all samples (30 repair scenarios [9 I-R-TD, 9 I-R-DI, 9 I-R-FC, 3 I-R-RE] and 30 production scenarios [9 I-P-MO, 9 I-P-RA, 9 I-P-FP, 3 I-P-RE]) are plotted in Figs. 4.24-4.31 to trace the impact of the contamination level (numbers $1,2,3)$ for a particular sample index $(-1$, $-2,-3)$, whereby the level of contamination increases as the level number increases. For the reference samples free from any contamination, the average values of $N(\mathrm{AV})$ were also calculated.

As shown in Figs. 4.24 and 4.25, the reference samples revealed the minimum values of $\mathrm{N} \approx 3$. The contamination of the adhesive noticeably increased the nonlinearity for all samples by 1.5-2 times. The maximum nonlinear ratio $\mathrm{N}$ was obtained for the sample TD 3-2 ( $\mathrm{N}=50 \pm 6$ [here and below in $10^{-3}$ units], outside the scale in Fig. 4.26). In this sample, the $N_{i}$ values were found to depend on the position of the measurement point. This fact, along with the anomalously high value of the 
Fig. 4.24 Non-linear ratios for the reference samples of the repair scenario
Fig. 4.25 Non-linear ratios for the reference samples of the production scenario
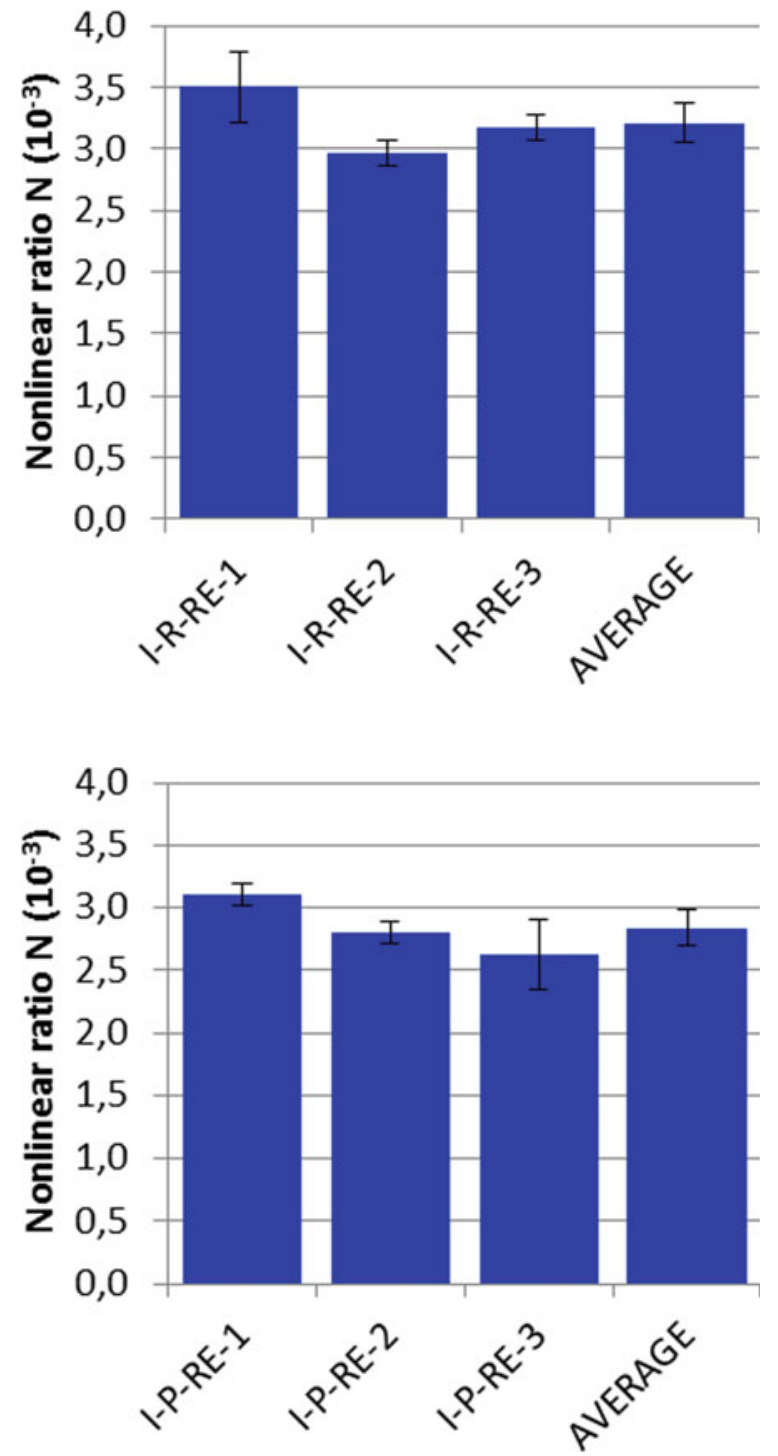

nonlinear ratio, indicates the presence of local delamination in the sample induced by thermal activation, which was also verified with conventional (linear) ultrasonic testing [8].

For the contamination types TD, RA, FP, DI, and FC, the material nonlinearity increased with the increase in the level of contamination (Figs. 4.26, 4.27, 4.28, 4.29, 4.30 , and 4.31). For each type of contamination, the values of $N$ changed noticeably with a variation of the contamination level, which indicates a sensitivity to the changes in the thin boundary layer between the adhesive and the adherends. According to the 
Fig. 4.26 Nonlinearity of each of the three samples $(-1,-2,-3)$ as a function of the TD level of contamination

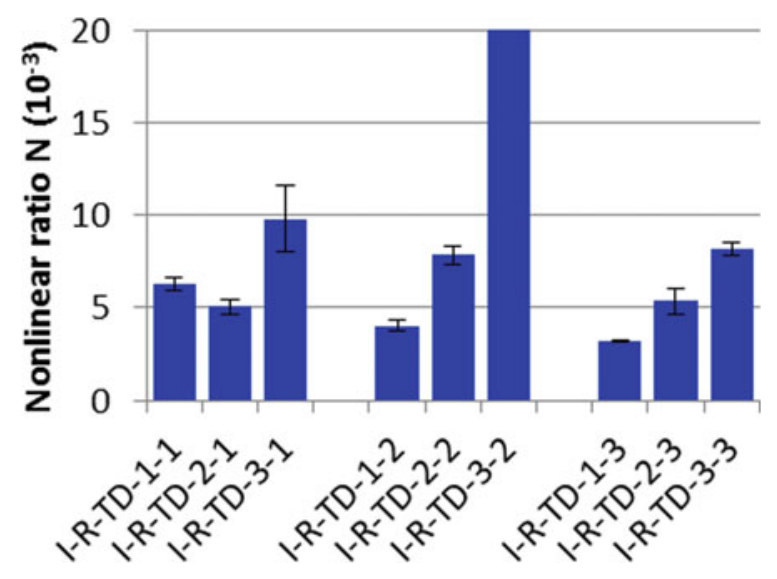

Fig. 4.27 Nonlinearity of each of the three samples $(-1,-2,-3)$ as a function of the RA level of contamination

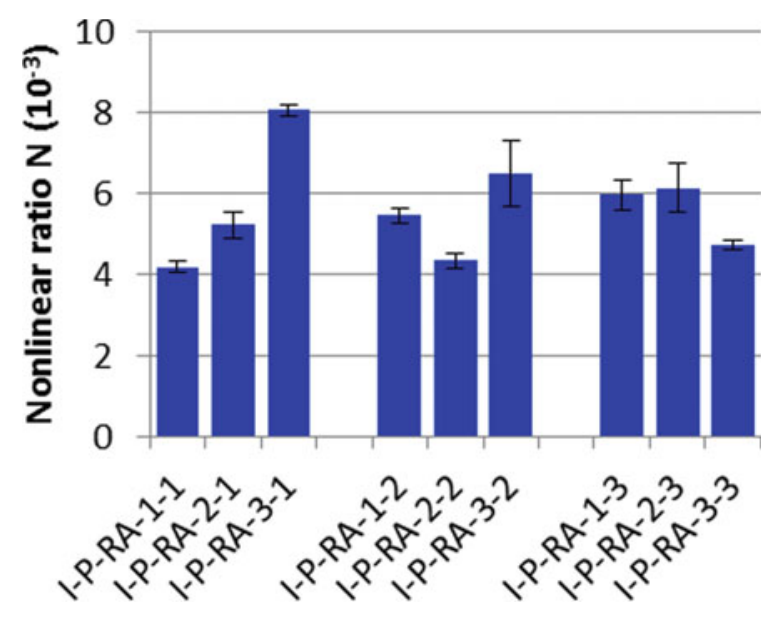

measurement results, the sensitivity of the nonlinear method is, therefore, sufficient to recognize the effect of contamination of the adhesive bonding.

The overall results for the nonlinearity variation for single contaminations are summarized in Figs. 4.32 and 4.33. Here, the average values of the nonlinear ratios are calculated for each group of samples with presumably the same level of contamination (groups 1,2,3) along with the average values for the reference samples. These data can be used to explicitly trace the nonlinearity behaviour as a function of the contamination level for each type, as well as between different types of contamination.

To correlate particular variations in $N$ with the strength of bonding as a function of the level of contamination, we refer back to the introduction to this chapter, where it was mentioned that the increase in nonlinearity is related to a "softening" or weakening of the material. A similar conclusion can be drawn based on the calculations of 
Fig. 4.28 Nonlinearity of each of the three samples $(-1,-2,-3)$ as a function of the DI level of contamination

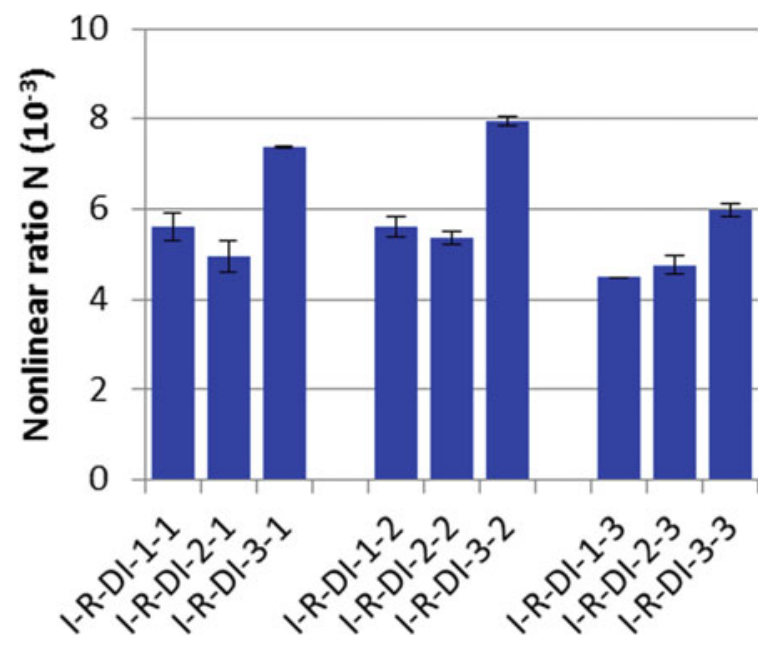

Fig. 4.29 Nonlinearity of each of the three samples $(-1,-2,-3)$ as a function of the FP level of contamination

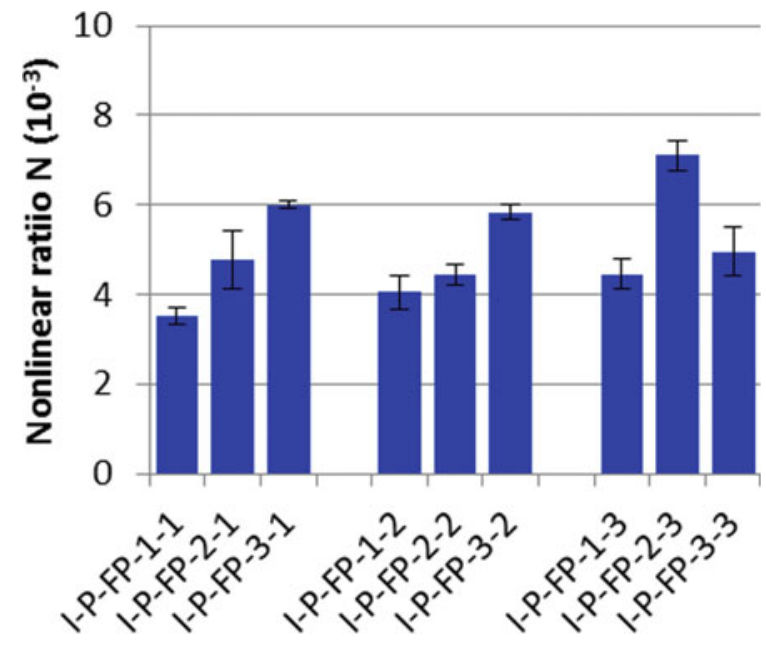

the stress-strain relations for the adhesive layer [30], which shows that this softening of the boundary layer increases its nonlinear response.

This fact goes some way towards explaining the lowest values $(N \approx 3)$ of the nonlinear ratios in the reference samples in Figs. 4.32 and 4.33; being free from any contamination, they are supposed to manifest the highest bonding strength. From this viewpoint, the strongest decrease in bonding strength was caused by the level 3 TD contamination (average value $\mathrm{N}=239$, outside the scale in Fig. 4.10). A similar (but somewhat lower) decrease in bonding strength was also recognized for the level 3 DI $(\mathrm{N}=7.10 .6)$, FP $(\mathrm{N}=5.60 .3)$, RA $(\mathrm{N}=6.40 .9)$, and FC $(\mathrm{N}=7.71 .6)$ contaminations (Figs. 4.9 and 4.10). The MO case is not so convincing as, with 


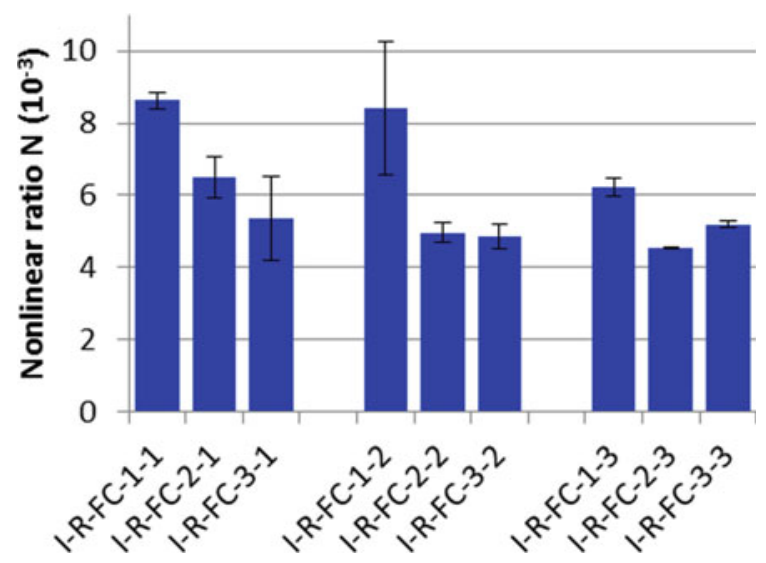

Fig. 4.30 Nonlinearity of each of the three samples $(-1,-2,-3)$ as a function of the FC level of contamination

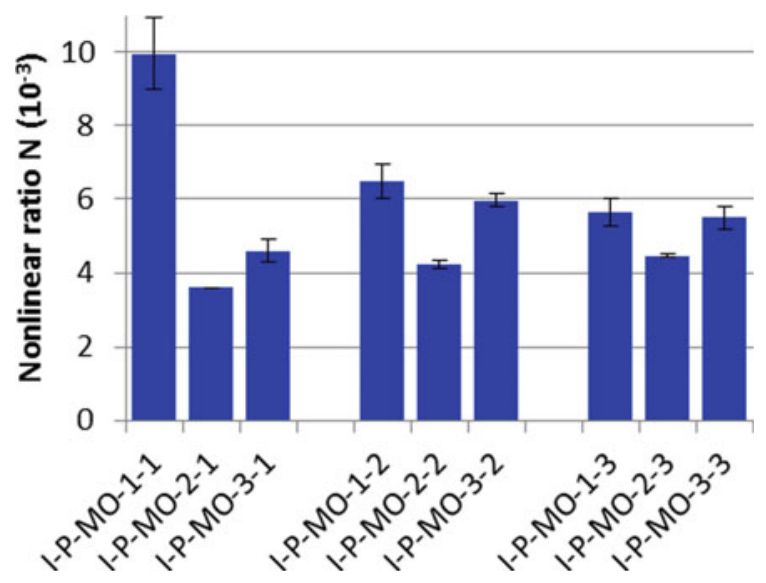

Fig. 4.31 Nonlinearity of each of the three samples $(-1,-2,-3)$ as a function of the MO level of contamination

the exception of an abrupt kick for sample MO1, the nonlinearity did not change noticeably, so $N \approx 5$ for all levels of contamination.

The effect of the combination of contaminations was studied for 12 multicontaminated flat CFRP samples, using two combinations of single contaminations:

- A constant low-intensity thermal degradation (TD1) with two different moderate amounts of de-icer (DI1, DI2) contamination applied to the surface prior to bonding.

- Two different amounts of release agent (RA1, RA2) and a constant high amount of fingerprint (FP3) contamination applied to the surface prior to bonding. 


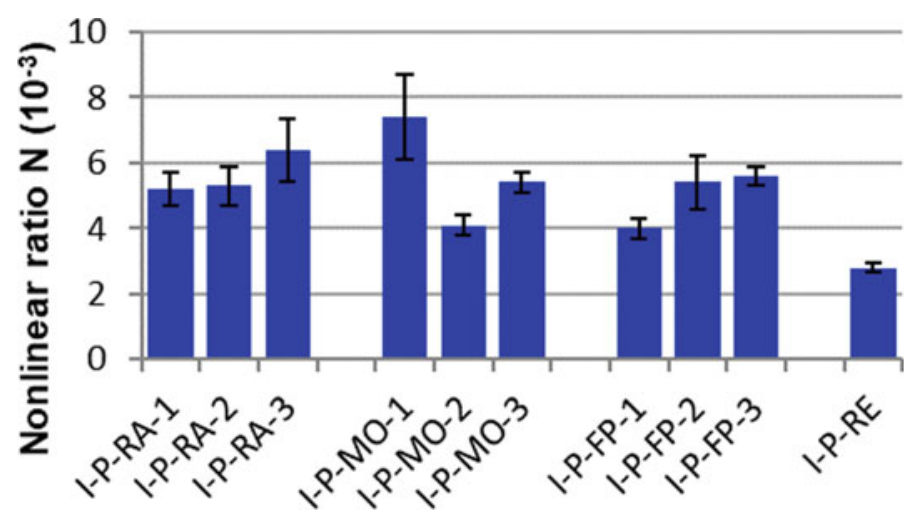

Fig. 4.32 Average nonlinear ratios as a function of the contamination levels for the samples of the production scenario

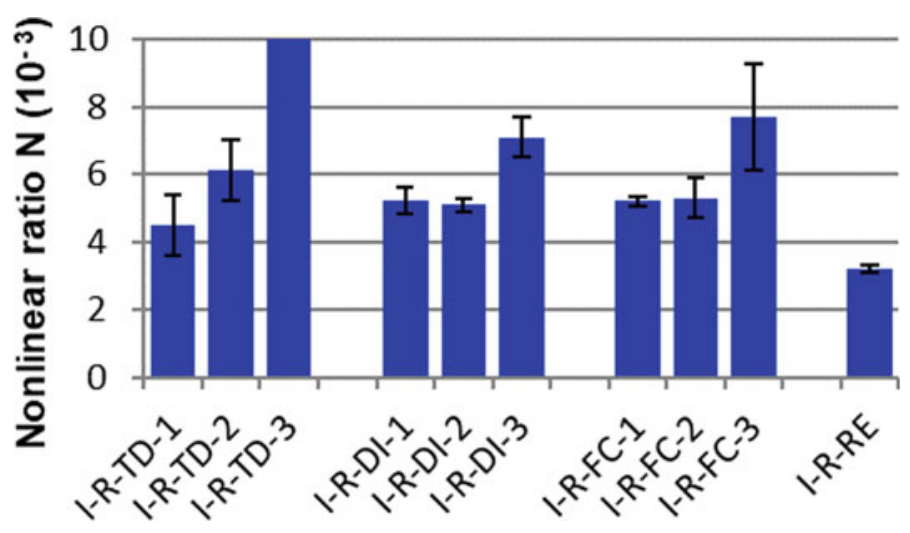

Fig. 4.33 Average nonlinear ratios as a function of the contamination levels for the samples of the repair scenario

The results of the measurements are shown in Figs. 4.34 and 4.35 along with the values of $N$ averaged separately over the groups with the same level of contamination (RA1 and RA2 in Fig. 4.34; DI1 and DI2 in Fig. 4.35). As shown in Fig. 4.34, the addition of a low amount of RA1 to highly contaminated FP3 samples had virtually no impact on the nonlinearity of the laminate, and the average value of $N$ for FP$3+\mathrm{RA}-1(5.8 \pm 0.4)$ stayed very close to the $N$ values that were previously measured for the single FP3 contaminated samples (5.6 \pm 0.3$)$, as shown in Fig. 4.32. However, the increase of the RA contamination level to RA2 enhanced the nonlinearity of the laminate noticeably, so that the average value $N$ for FP-3+RA-2 in Fig. 4.34 is $6.9 \pm 0.4$.

A similar effect of the increase of nonlinearity was observed when increasing the level of DI contamination in combination with TD-1 (Fig. 4.35). The addition 
Fig. 4.34 Nonlinearity of contaminated FP3 samples $(-1,-2,-3)$ additionally affected by two levels of RA (RA1 and RA2) contaminations

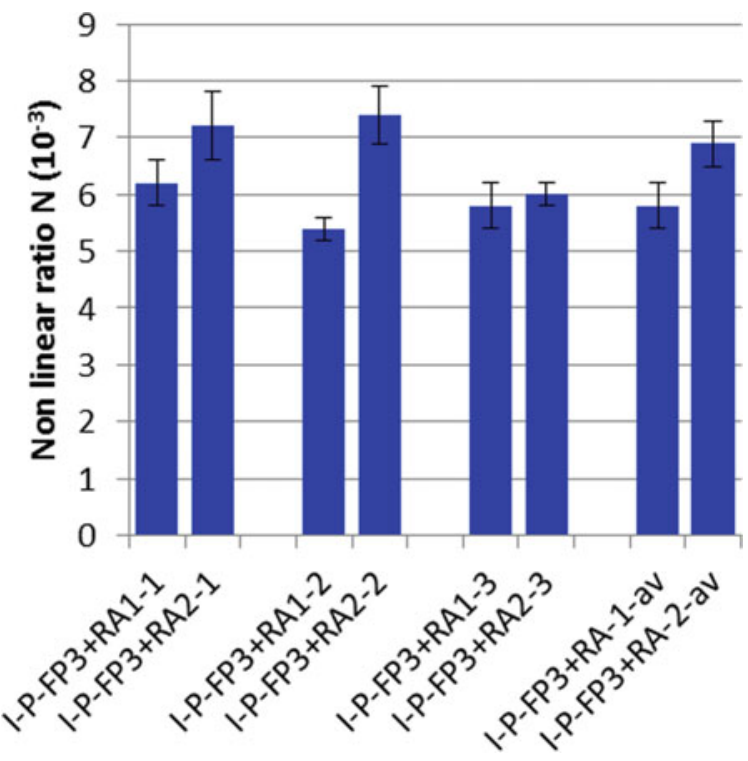

Fig. 4.35 Nonlinearity of contaminated TD1 samples $(-1,-2,-3)$ additionally affected by two levels of DI (DI1 and DI2) contaminations

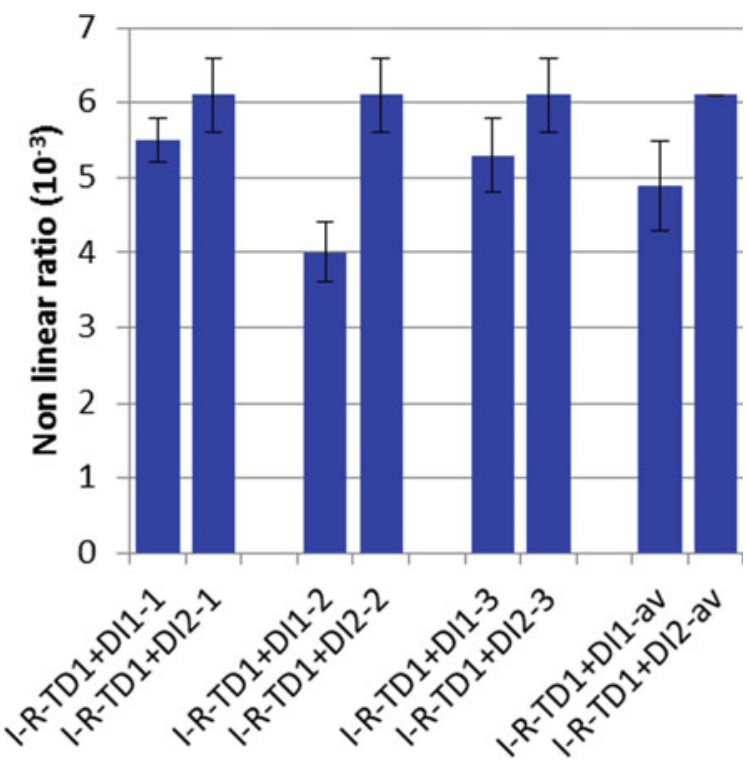

of DI-1 left the average value of $\mathrm{N}$ for TD-1 (4.5 \pm 0.9 ; see Fig. 4.33) unchanged within the margin of error, namely $4.9 \pm 0.6$ (Fig. 4.35). A moderate level of DI-2 enhanced the nonlinearity level of the laminate substantially, whereby the N for DI2+TD-1 increased to 6.1 (Fig. 4.35). Both combinations, therefore, demonstrated a 
cumulative decline in the adhesion between flat coupons caused by the increase of the DI and RA contaminations.

\subsubsection{Nonlinear Ultrasonic Technique Results on Pilot Level Samples}

The nine scarfed samples studied comprised three reference samples (without contamination) and samples with a low level of thermal degradation (TD-1) and two different levels of de-icer contamination (DI-1 and DI-2) applied to the surface of the laminates prior to bonding. These samples were glued at a slant in an attempt to mimic the repairing of composite components in practice. The results of the measurements are presented in Fig. 4.36.

For two sets out of the three samples measured, the experimental data demonstrated an increase in the nonlinearity caused by the contamination in comparison to the reference samples (sets 1 and 3; Fig. 4.36). The nonlinearity of sample set 2 did not change within the margin of error. A particularly strong effect of contamination was observed for sample set 3 (approx. 150\% increase in nonlinearity). The effect of the increase of the level of DI contamination was not as noticeable, as was also the case in the adhesion of conventional flat laminates (see Fig. 4.33), whereby the average $N$ values practically did not change between DI-1 and DI-2 (Fig. 4.36). This behaviour shows that even a moderate amount of DI leads to major deterioration of the adhesive properties, while a further increase of the contamination level does not substantially further affect the bonding strength.

Fig. 4.36 Nonlinearity of three sets of scarfed samples: reference and TD1 $(-1,-2$, -3 ) affected by two levels of DI (DI1 and DI2) contaminations

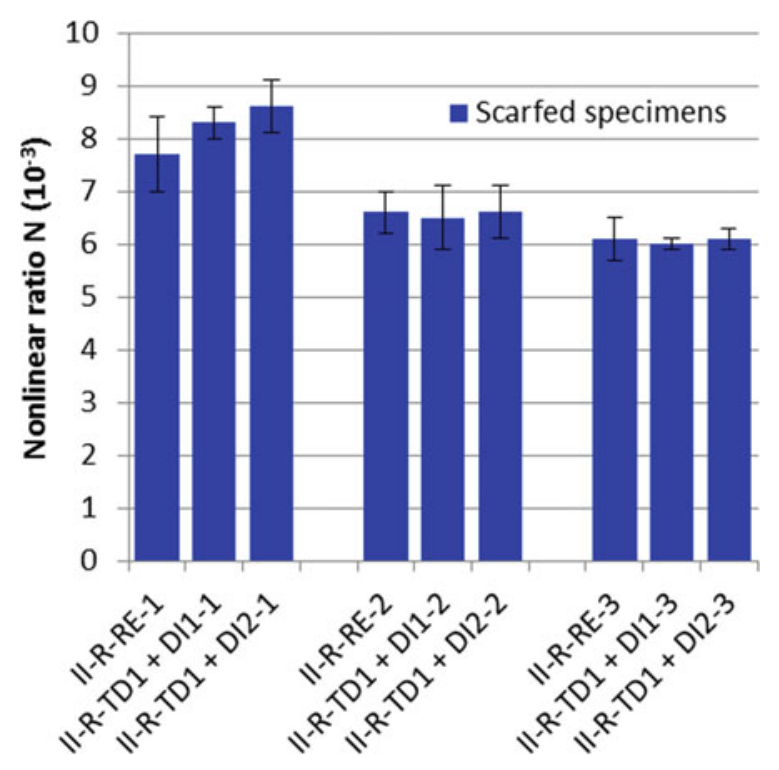


Fig. 4.37 Nonlinearity of three sets of curved samples: reference and FP3 $(-1,-2$, -3 ) affected by two levels of RA (RA1 and RA2) contaminations

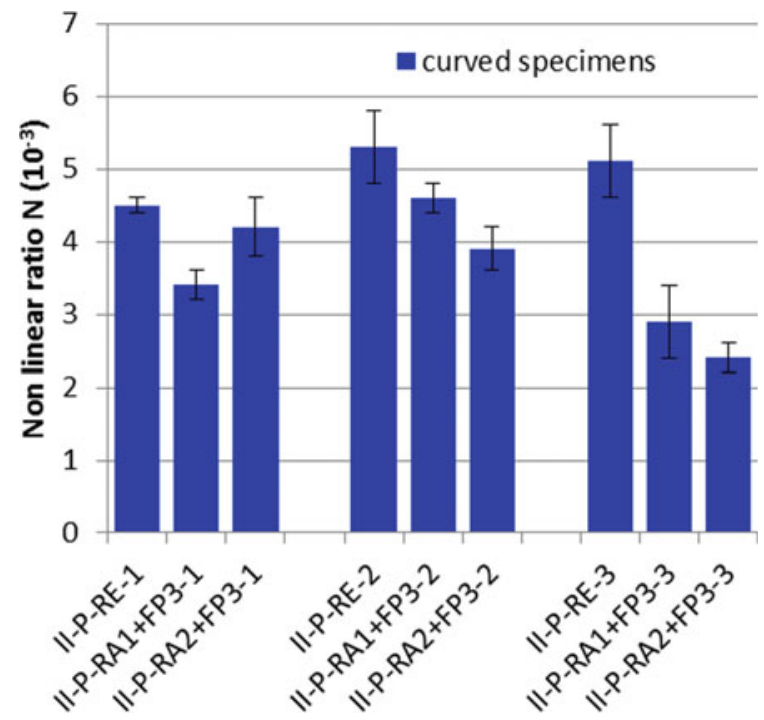

The nine production samples (curved) comprised three reference samples (without contamination) and six samples with a constant high level of fingerprint contamination (FP-3) along with two different moderate levels of release agent (RA-1 and RA-2) applied to the surface of the laminates prior to bonding. The results of the measurements are shown in Fig. 4.37.

All three sets of curved samples reliably indicated a decrease in the nonlinearity (and therefore, a strengthening of the bonds) induced by the contamination, whereby the highest values of $N$ were observed for the reference samples. For sample sets 2 and 3 , the decrease caused by the contamination resulted in very low values comparable to those measured for flat non-contaminated samples. Unlike similarly contaminated flat coupons (Fig. 4.34), the nonlinearity further decreased (strengthening of the bonds) as soon as the contamination level increased from RA-1 to RA-2. An interpretation of this unconventional behaviour would need to involve more detailed information on the technology and process of adhesive bonding for the curved sample, including data on the internal stresses in the laminate.

\subsection{Conclusion}

In this chapter, we highlight the relevance and performance achieved when applying extended non-destructive testing (ENDT) methods for the bond line quality assessment of adhesive joints. For important application scenarios with regard to aircraft manufacture or the in-service repair of composite structures, the in-process monitoring of CFRP composites is facilitated through the use of advanced setups and approaches based on electromechanical impedance (EMI), laser shock adhesion 
testing (LASAT), and the nonlinear ultrasound method (NUS). Adhesive joints in the shape of flat coupons, scarfed samples or curved samples were investigated and correlations with the results from assessing design-relevant joint features were demonstrated for the obtained ENDT datasets.

The EMI method enabled differentiation of the reference samples from the contaminated samples in the production scenario. This was possible for the flat samples. In case of moisture contamination, the increasing frequency shift correlates with the contamination level. Due to the different geometry of the curved samples, another bandwidth was analyzed and a clear differentiation between the reference and contaminated samples was not possible. In the case of the repair scenario, this technique enabled the detection of almost all the flat samples with modifications. The numerical parameter for comparison was defined after inspecting the spectrum of each sample and also of the free sensors. In the case of thermal degradation and faulty curing, the highest level of modification was easily detected. This was also possible for the combined contamination case. In the case of scarfed samples, the detection of four out of five samples was possible. However, the sensitivity to the level of contamination was not obvious.

The LASAT gave good results for the coupon samples, and these are in accordance with results found during the $\mathrm{G}_{\mathrm{IC}}$ and $\mathrm{G}_{\mathrm{IIC}}$ tests. It was also possible in certain cases to identify the different levels of contamination. The LASAT did not differentiate the samples from the production scenario with multiple contaminations from the reference sample. The intensity required to open the faulty joints was the same as that for the reference. The results are also hard to explain for the repair scenario, where the technology detected the least contaminated sample but was not able to clearly detect the most contaminated sample. However, the same contamination on scarfed samples showed much better results, whereby the LASAT technology was effective at differentiating a sound bond from a weak one. The difference between the results of the coupon samples and those of the scarfed samples is not yet fully understood. The bond geometry may have played a part in the crack detection/propagation within the contaminated samples. Furthermore, this technology heavily relies on the standard sample, whereby the reference used for the coupon samples may have been produced with different parameters. Further studies need to be conducted in order to draw conclusions from the results for these samples. The curved samples were also highly porous and did not follow the specifications. In summary, no relevant conclusion can be drawn from these particular results.

The nonlinear measurements revealed an increase in the nonlinearity caused by contamination of the adhesive layer for most single contamination types. For each contamination type, the values of the nonlinearity ratio $N$ changed noticeably with a variation of the contamination level, which indicates a sensitivity to the changes in the thin boundary layer between the adhesive and the adherends. According to the measurement results, the sensitivity of the nonlinear method is, therefore, sufficient to recognize the effect of contamination on adhesive bonding. For the contaminations types TD, RA, FP, DI, and FC, the material nonlinearity increased with the increase of the level of contamination. Since nonlinearity increases due to a "softening" of the material, an increase in $N$ is an indication of a weakening of the adhesive bond. 
The effect of multiple contaminations was studied for the FP3+RA and TD1+DI contaminations. Both combinations demonstrate a cumulative decline of the adhesion between flat samples caused by the increase of the DI and RA contaminations. In scarfed samples, the effects of multiple contaminations TD1+DI similarly led to an increase in nonlinearity, which indicates a decline in bond quality. However, the effect of an increase in the level of DI contamination was not as noticeable, as was the case in the bonding of conventional flat samples. This behaviour shows that even a moderate amount of DI leads to major deterioration of the adhesive properties, while a further increase of the contamination level does not affect the bonding strength substantially. The effect of multiple contaminations offers a somewhat different result for curved CFRP laminates, whereby all three sets of curved samples studied reliably indicated a decrease of nonlinearity (and therefore a strengthening of the bond) induced by the contamination. The interpretation of this unconventional behaviour should involve detailed information on the technology and the process of adhesive bonding for curved samples, including data on the internal stresses in the laminate.

In summary, our investigations reveal that in certain scenarios the ENDT methods EMI, LASAT, and NUS are sensitive to impacts affecting a bond strength reduction and can, therefore, be utilized for identifying not in order (NIO) joints during bond line assessment. In the final research chapter of this book, we will underline this perception and prognosis with findings highlighting the performance of ENDT for the monitoring of quality-relevant operand features in adhesive bonding processes involving parts of real aerospace structures with stringers.

\section{References}

1. Wadley HNG (1988) Interfaces: the next NDE challenge. In: Thompson DO, Chimenti DE (eds) Review of progress in quantitative nondestructive evaluation: volume 7B. vol 42. Springer US, Boston, MA, pp 881-892

2. ENCOMB "Extended Non-Destructive Testing of Composite Bonds" (2010-2014) European Seventh Framework Programme for research and technological development (FP7). Grant agreement ID: 266226

3. ComBoNDT "Quality assurance concepts for adhesive bonding of aircraft composite structures by advanced NDT" (2015-2018) Horizon 2020; research and innovation programme (H2020). Grant agreement ID: 636494

4. Dugnani R, Zhuang Y, Kopsaftopoulos F et al (2016) Adhesive bond-line degradation detection via a cross-correlation electromechanical impedance-based approach. Struct Health Monit 15(6):650-667. https://doi.org/10.1177/1475921716655498

5. Dugnani R, Chang F-K (2017) Analytical model of lap-joint adhesive with embedded piezoelectric transducer for weak bond detection. J Intell Mater Syst Struct 28(1):124-140. https:// doi.org/10.1177/1045389X16645864

6. Malinowski P, Wandowski T, Ostachowicz W (2015) The use of electromechanical impedance conductance signatures for detection of weak adhesive bonds of carbon fibre-reinforced polymer. Struct Health Monit 14(4):332-344. https://doi.org/10.1177/1475921715586625

7. Malinowski PH, Tserpes KI, Ecault R et al (2017) Study of adhesive bonds by mechanical tests, ultrasounds and electromechanical impedance method. In: Structural health monitoring 2017: real-time material state awareness and data-driven safety assurance. Proceedings of the 
eleventh international workshop on structural health monitoring, Stanford University, Stanford, CA, September 12-14, 2017. DEStech Publications, Inc, Lancaster, PA

8. Malinowski PH, Ecault R, Wandowski T et al (2017) Evaluation of adhesively bonded composites by nondestructive techniques. In: Kundu $\mathrm{T}$ (ed) Health monitoring of structural and biological systems 2017. SPIE, 101700B

9. Berthe L, Fabbro R, Peyre P et al (1997) Shock waves from a water-confined laser-generated plasma. J Appl Phys 82(6):2826-2832. https://doi.org/10.1063/1.366113

10. Fabbro R, Fournier J, Ballard P et al (1990) Physical study of laser-produced plasma in confined geometry. J Appl Phys 68(2):775-784. https://doi.org/10.1063/1.346783

11. Vossen JL (1978) Measurements of film-substrate bond strength by laser spallation. In: Mittal KL (ed) Adhesion measurement of thin films, thick films, and bulk coatings. ASTM International, 100 Barr Harbor Drive, PO Box C700, West Conshohocken, PA 19428-2959, $122-122-12$

12. Yuan J, Gupta V, Pronin A (1993) Measurement of interface strength by the modified laser spallation technique. III. Experimental optimization of the stress pulse. J Appl Phys 74(4):24052410. https://doi.org/10.1063/1.354700

13. Boustie M, Auroux E, Romain J-P et al (1999) Determination of the bond strength of some microns coatings using the laser shock technique. Eur Phys J AP 5(2):149-153. https://doi.org/ 10.1051/epjap:1999123

14. Bolis C, Berthe L, Boustie M et al (2007) Physical approach to adhesion testing using laserdriven shock waves. J Phys D Appl Phys 40(10):3155-3163. https://doi.org/10.1088/00223727/40/10/019

15. Gilath I, Eliezer S, Bar-Noy T et al (1993) Material response at hypervelocity impact conditions using laser induced shock waves. Int J Impact Eng 14(1-4):279-289. https://doi.org/10.1016/ 0734-743X(93)90027-5

16. Bossi R, Housen K, Walters C, Works BP (2005) Laser bond inspection device for composites: has the holy grail been found. NTIAC Newslett 30

17. Ecault R, Berthe L, Touchard Fet al (2015) Experimental and numerical investigations of shock and shear wave propagation induced by femtosecond laser irradiation in epoxy resins. J Phys D Appl Phys 48(9):95501. https://doi.org/10.1088/0022-3727/48/9/095501

18. Gay E, Berthe L, Boustie M et al (2014) Study of the response of CFRP composite laminates to a laser-induced shock. Compos B Eng 64:108-115. https://doi.org/10.1016/j.compositesb. 2014.04.004

19. Courapied D, Berthe L, Peyre P et al (2015) Laser-delayed double shock-wave generation in water-confinement regime. J Laser Appl 27(S2):S29101. https://doi.org/10.2351/1.4906382

20. Chen J, Nakamura T, Aoki $\mathrm{K}$ et al (2001) Curing of epoxy resin contaminated with water. J Appl Polym Sci 79(2):214-220. https://doi.org/10.1002/1097-4628(20010110)79:2\%3c214: AID-APP30\%3e3.0.CO;2-S

21. Laporte D (2011) Analyse de la réponse d'assemblages collés sous des sollicitations en dynamique rapide, Chasseneuil-du-Poitou, Ecole nationale supérieure de mécanique et d'aérotechnique

22. Moutsompegka E, Tserpes KI, Polydoropoulou P et al (2017) Experimental study of the effect of pre-bond contamination with de-icing fluid and ageing on the fracture toughness of composite bonded joints. Fatigue Fract Engng Mater Struct 40(10):1581-1591. https://doi.org/10.1111/ ffe. 12660

23. Brenzeale MA, Philip J (1984) Determination of third-order elastic constants from ultrasonic harmonic generation measurement. Phys Acoust XVII

24. Guyer RA, Johnson PA (2009) Nonlinear mesoscopic elasticity: the complex behaviour of granular media including rocks and soil. Wiley-VCH, Weinheim

25. Solodov IY, Krohn N, Busse G (2002) CAN: an example of nonclassical acoustic nonlinearity in solids. Ultrasonics 40(1-8):621-625. https://doi.org/10.1016/S0041-624X(02)00186-5

26. Nagy PB (1998) Fatigue damage assessment by nonlinear ultrasonic materials characterization. Ultrasonics 36(1-5):375-381. https://doi.org/10.1016/S0041-624X(97)00040-1 
27. Zheng Y, Maev RG, Solodov IY (2000) Review/Sythèse nonlinear acoustic applications for material characterization: a review. Can J Phys 77(12):927-967. https://doi.org/10.1139/ p99-059

28. Pruell C, Kim J-Y, Qu J et al (2009) Evaluation of fatigue damage using nonlinear guided waves. J Appl Phys 18(3):35003. https://doi.org/10.1088/0964-1726/18/3/035003

29. Zhao J, Chillara VK, Ren B et al (2016) Second harmonic generation in composites: theoretical and numerical analyses. J Appl Phys 119(6):64902. https://doi.org/10.1063/1.4941390

30. Li W, Cho Y, Achenbach JD (2012) Detection of thermal fatigue in composites by second harmonic Lamb waves. Smart Mater Struct 21(8):85019. https://doi.org/10.1088/0964-1726/ 21/8/085019

Open Access This chapter is licensed under the terms of the Creative Commons Attribution 4.0 International License (http://creativecommons.org/licenses/by/4.0/), which permits use, sharing, adaptation, distribution and reproduction in any medium or format, as long as you give appropriate credit to the original author(s) and the source, provide a link to the Creative Commons license and indicate if changes were made.

The images or other third party material in this chapter are included in the chapter's Creative Commons license, unless indicated otherwise in a credit line to the material. If material is not included in the chapter's Creative Commons license and your intended use is not permitted by statutory regulation or exceeds the permitted use, you will need to obtain permission directly from the copyright holder.

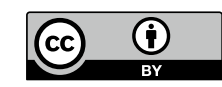

